\title{
Inertial Force Coupling to Nonlinear Aeroelasticity of Flexible Wing Aircraft
}

\author{
Nhan Nguyen * \\ NASA Ames Research Center, Moffett Field, CA 94035 \\ Eric Ting ${ }^{\dagger}$ \\ Stinger Ghaffarian Technologies, Inc., Moffett Field, CA 94035
}

\begin{abstract}
This paper investigates the inertial force effect on nonlinear aeroelasticity of flexible wing aircraft. The geometric are nonlinearity due to rotational and tension stiffening. The effect of large bending deflection will also be investigated. Aeroelastic analysis will be conducted for a truss-braced wing aircraft concept with nonlinear effect of large bending deflection and inertial force coupling.
\end{abstract}

\section{Introduction}

Modern aircraft are increasingly designed to be highly maneuverable in order to achieve high-performance mission objectives. Toward this goal, aircraft designers have been adopting light-weight, flexible, high aspect ratio wings in modern aircraft. Aircraft design concepts that take advantage of wing flexibility to increase aerodynamic performance and maneuverability have been investigated. By twisting a wing structure, an aerodynamic moment can be generated to enable an aircraft to execute a maneuver in place of the use of traditional control surfaces. For example, a rolling moment can be induced by twisting the left and right wings in the opposite direction. Similarly, a pitching moment can be generated by twisting both wings in the same direction. Wing twisting or warping for flight control is not a new concept and was used in the Wright Flyer in the 1903. The U.S. Air Force conducted the Active Flexible Wing program in the 1980's and 1990's to explore potential use of leading edge slats and trailing edge flaps to increase control effectiveness of F-16 aircraft for high speed maneuvers. ${ }^{1}$ In the recent years, the Active Aeroelastic Wing research program also investigated a similar technology to induce wing twist in order to improve roll maneuverability of F/A18 aircraft. $^{2}$ Wing shaping control concepts for drag reduction are being studied by NASA to leverage wing flexibility for aerodynamic performance. ${ }^{3,4}$ By re-twisting a flexible wing and using variable camber aerodynamic control surfaces, aircraft wings can have a mission-adaptive capability. ${ }^{5}$ In recognition of the role of aeroelasticity on aircraft performance and dynamics, NASA Advanced Air Transport Technology (AATT) project is conducting research in the area of Performance Adaptive Aeroelastic Wing (PAAW). This research develops concepts such as the variable camber continuous trailing edge flap (VCCTEF) to enable wing shaping control for aerodynamic performance and dynamics. ${ }^{6}$

Structural deflections of lifting surfaces interact with aerodynamic forces to create aeroelastic coupling that can affect aircraft performance. Understanding these effects can improve the prediction of aircraft flight dynamics and can provide insight into how to design a flight control system that can reduce aeroelastic interactions with a rigid-body flight controller. Generally, high aspect ratio lifting surfaces undergo a greater degree of structural deflections than low aspect ratio lifting surfaces. As a result, the natural frequencies of the aeroelastic modes decrease as the aspect ratio increases. This may reduce the desired frequency separation between flexible wing aeroelastic modes and aircraft rigid-body modes that could potentially cause undesirable flight control interactions. For example, when a pilot commands a roll maneuver, the aileron deflections can cause one or more aeroelastic wing modes to excite. The aeroelastic modes can result in changes to the intended aerodynamics of the wings, thereby potentially causing undesired aircraft

\footnotetext{
*NASA Ames Research Center, Research Scientist, AIAA Associate Fellow, nhan.t.nguyen@nasa.gov

†Stinger Ghaffarian Technologies Inc., NASA Ames Research Center, Research Engineer, eric.b.ting@nasa.gov
} 
responses. Aeroservoelastic filtering is a traditional method for suppressing elastic modes, but this usually comes at an expense in terms of reducing the phase margin in a flight control system. If the phase margin is significantly reduced, aircraft responses may become more sluggish to pilot commands. Consequently, with a phase lag in the control inputs, potential pilot-induced oscillations (PIOs) can occur. Numerous studies have been made to increase the understanding of the role of aeroservoelasticity in the design of flight control systems.

Due to the flexibility of modern aircraft structures, flight dynamic models of rigid-body aircraft have limitations and cannot accurately predict behaviors of flexible aircraft when aeroelastic modes participate in the rigid-body motion. Recently, some investigators have investigated theoretical approaches to developing integrated flight dynamics with aeroelasticity. Shearer develops an integrated flight dynamic model for a representative High Altitude Long Endurance (HALE) vehicle. To fully account for the multi-physics interactions that normally exist in a flexible aircraft, inertial-aeroelastic-propulsive force coupling must be addressed in aircraft aeroelasticity. ${ }^{7}$

The purpose of this study is to investigate the inertial force effect on aircraft elasticity with geometric nonlinearity due to rotational and tension stiffening. The effect of large bending deflection will also be investigated. Flutter analysis will be conducted for a truss-braced wing aircraft concept with tension stiffening.

\section{Inertial Force Analysis}

Consider an airfoil section on the left wing as shown in Fig. 1 undergoing bending and torsional deflections. Let $(x, y, z)$ be the undeformed coordinates of point $\mathrm{Q}$ on a wing airfoil section in the reference frame $\mathrm{D}$ defined by unit vectors $\left(\mathbf{d}_{1}, \mathbf{d}_{2}, \mathbf{d}_{3}\right)$. Let $\mathbf{p}_{0}=x \mathbf{d}_{1}$ be a position vector along the elastic axis. Then, point $\mathrm{Q}$ is defined by a position vector $\mathbf{p}=\mathbf{p}_{0}+\mathbf{q}$ where $\mathbf{q}=y \mathbf{d}_{2}+z \mathbf{d}_{3}$ defines point $\mathrm{Q}$ in the $y-z$ plane from the elastic axis. Then the undeformed local airfoil coordinates of point $\mathrm{Q}$ are

$$
\left[\begin{array}{l}
y \\
z
\end{array}\right]=\left[\begin{array}{cc}
\cos \gamma & -\sin \gamma \\
\sin \gamma & \cos \gamma
\end{array}\right]\left[\begin{array}{l}
\eta \\
\xi
\end{array}\right]
$$

where $\eta$ and $\xi$ are local airfoil coordinates, and $\gamma$ is the wing section pre-twist angle, positive nose-down. ${ }^{8,9}$

Differentiating $y$ and $z$ with respect to $x$ gives

$$
\left[\begin{array}{l}
y_{x} \\
z_{x}
\end{array}\right]=\gamma^{\prime}\left[\begin{array}{cc}
-\sin \gamma & -\cos \gamma \\
\cos \gamma & -\sin \gamma
\end{array}\right]\left[\begin{array}{l}
\eta \\
\xi
\end{array}\right]=\left[\begin{array}{c}
-z \gamma^{\prime} \\
y \gamma^{\prime}
\end{array}\right]
$$
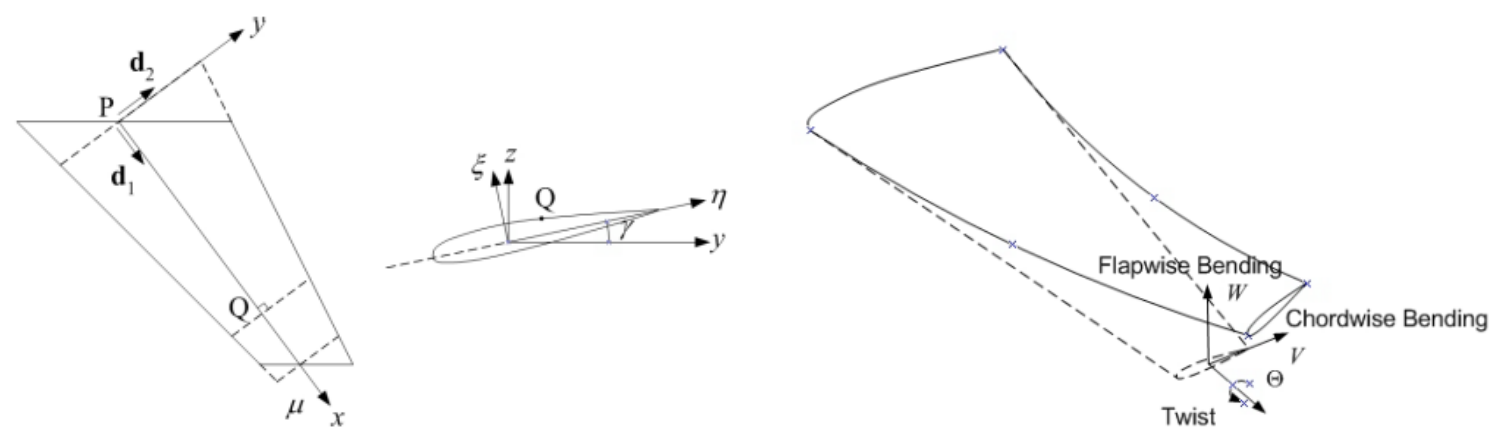

Figure 1. Left Wing Reference Frame of Wing in Combined Bending-Torsion

Let $\Theta$ be a torsional twist angle about the $x$-axis, positive nose-down. Let $W$ and $V$ be flapwise and chordwise bending deflections of point $\mathrm{Q}$, respectively. Let $U$ be the axial displacement of point $\mathrm{Q}$. Then, the displacement and rotation vectors due to the elastic deformation can be expressed as

$$
\mathbf{r}=U \mathbf{d}_{1}+V \mathbf{d}_{2}+W \mathbf{d}_{3}
$$




$$
\phi=\Theta \mathbf{d}_{1}-W_{x} \mathbf{d}_{2}+V_{x} \mathbf{d}_{3}
$$

where the subscripts $x$ and $t$ denote the partial derivatives of $\Theta, W$, and $V$.

Let $\left(x_{1}, y_{1}, z_{1}\right)$ be the deformed coordinates of point $\mathrm{Q}$ on the airfoil in the reference frame $\mathrm{D}$ and $\mathbf{p}_{1}=x_{1} \mathbf{d}_{1}+y_{1} \mathbf{d}_{2}+z_{1} \mathbf{d}_{3}$ be its position vector. Then the coordinates $\left(x_{1}, y_{1}, z_{1}\right)$ are computed as

$$
\mathbf{p}_{1}=\mathbf{p}+\mathbf{r}+\phi \times \mathbf{q}
$$

where

$$
\left[\begin{array}{l}
x_{1} \\
y_{1} \\
z_{1}
\end{array}\right]=\left[\begin{array}{c}
x+U-y V_{x}-z W_{x} \\
y+V-z \Theta \\
z+W+y \Theta
\end{array}\right]
$$

Let $\overline{\mathbf{v}}=u \mathbf{b}_{1}+v \mathbf{b}_{2}+w \mathbf{b}_{3}$ be the aircraft velocity vector at the aircraft center of gravity (CG) where $\left(\mathbf{b}_{1}, \mathbf{b}_{2}, \mathbf{b}_{3}\right)$ are the unit vectors in aircraft body-fixed reference frame $\mathrm{B}$ in the roll, pitch, and yaw axes according to the standard aircraft convention. Let $\boldsymbol{\omega}=p \mathbf{b}_{1}+q \mathbf{b}_{2}+r \mathbf{b}_{3}$ be the aircraft angular velocity vector where $(p, q, r)$ are aircraft angular velocity components in the roll, pitch, and yaw axes. Let $\mathbf{r}_{a}=$ $-x_{a} \mathbf{b}_{1}-y_{a} \mathbf{b}_{2}-z_{a} \mathbf{b}_{3}$ be the position vector of point $\mathrm{Q}$ in the aircraft body-fixed reference frame $\mathrm{B}$ relative to the aircraft $\mathrm{CG}$ such that $x_{a}$ is positive when point $\mathrm{Q}$ is aft of the aircraft $\mathrm{CG}, y_{a}$ is positive when point $\mathrm{Q}$ is toward the left wing from the aircraft $\mathrm{CG}$, and $z_{a}$ is positive when point $\mathrm{Q}$ is above the aircraft CG. The velocity at point $\mathrm{Q}$ due to the aircraft velocity and angular velocity in the reference frame $\mathrm{D}$ is then computed as

$$
\begin{aligned}
\mathbf{v}_{Q}=\overline{\mathbf{v}}+\boldsymbol{\omega} \times \mathbf{r}_{a} & =\left(u \mathbf{b}_{1}+v \mathbf{b}_{2}+w \mathbf{b}_{3}\right)+\left(p \mathbf{b}_{1}+q \mathbf{b}_{2}+r \mathbf{b}_{3}\right) \times\left(-x_{a} \mathbf{b}_{1}-y_{a} \mathbf{b}_{2}-z_{a} \mathbf{b}_{3}\right) \\
& =\left(u+r y_{a}-q z_{a}\right) \mathbf{b}_{1}+\left(v-r x_{a}+p z_{a}\right) \mathbf{b}_{2}+\left(w+q x_{a}-p y_{a}\right) \mathbf{b}_{3}=x_{t} \mathbf{d}_{1}+y_{t} \mathbf{d}_{2}+z_{t} \mathbf{d}_{3}
\end{aligned}
$$

where

$$
\left[\begin{array}{c}
x_{t} \\
y_{t} \\
z_{t}
\end{array}\right]=\left[\begin{array}{c}
-\left(u+r y_{a}-q z_{a}\right) \sin \Lambda \cos \Gamma-\left(v-r x_{a}+p z_{a}\right) \cos \Lambda \cos \Gamma-\left(w+q x_{a}-p y_{a}\right) \sin \Gamma \\
-\left(u+r y_{a}-q z_{a}\right) \cos \Lambda+\left(v-r x_{a}+p z_{a}\right) \sin \Lambda \\
\left(u+r y_{a}-q z_{a}\right) \sin \Lambda \sin \Gamma+\left(v-r x_{a}+p z_{a}\right) \cos \Lambda \sin \Gamma-\left(w+q x_{a}-p y_{a}\right) \cos \Gamma
\end{array}\right]
$$

The transformation between $\left(\mathbf{b}_{1}, \mathbf{b}_{2}, \mathbf{b}_{3}\right)$ and $\left(\mathbf{d}_{1}, \mathbf{d}_{2}, \mathbf{d}_{3}\right)$ is given by

$$
\left[\begin{array}{l}
\mathbf{b}_{1} \\
\mathbf{b}_{2} \\
\mathbf{b}_{3}
\end{array}\right]=\left[\begin{array}{ccc}
-\sin \Lambda \cos \Gamma & -\cos \Lambda & \sin \Lambda \sin \Gamma \\
-\cos \Lambda \cos \Gamma & \sin \Lambda & \cos \Lambda \sin \Gamma \\
-\sin \Gamma & 0 & -\cos \Gamma
\end{array}\right]\left[\begin{array}{l}
\mathbf{d}_{1} \\
\mathbf{d}_{2} \\
\mathbf{d}_{3}
\end{array}\right]
$$

The local velocity at point $\mathrm{Q}$ due to aircraft rigid-body dynamics and aeroelastic deflections in the reference frame $\mathrm{D}$ is obtained as $^{9}$

$$
\mathbf{v}=\mathbf{v}_{Q}+\frac{\partial \Delta \mathbf{p}}{\partial t}+(\boldsymbol{\omega}+\dot{\phi}) \times \Delta \mathbf{p}=v_{x} \mathbf{d}_{1}+v_{y} \mathbf{d}_{2}+v_{z} \mathbf{d}_{3}
$$

where $\Delta \mathbf{p}=\mathbf{p}_{1}-\mathbf{p}$ and

$$
\begin{gathered}
{\left[\begin{array}{c}
v_{x} \\
v_{y} \\
v_{z}
\end{array}\right]=\left[\begin{array}{c}
x_{t}-\left(\omega_{z}+V_{x t}\right)(V-z \Theta)+\left(\omega_{y}-W_{x t}\right)(W+y \Theta)+U_{t}-y V_{x t}-z W_{x t} \\
y_{t}+\left(\omega_{z}+V_{x t}\right)\left(U-y V_{x}-z W_{x}\right)-\left(\omega_{x}+\Theta_{t}\right)(W+y \Theta)+V_{t}-z \Theta_{t} \\
z_{t}-\left(\omega_{y}-W_{x t}\right)\left(U-y V_{x}-z W_{x}\right)+\left(\omega_{x}+\Theta_{t}\right)(V-z \Theta)+W_{t}+y \Theta_{t}
\end{array}\right]} \\
{\left[\begin{array}{c}
\omega_{x} \\
\omega_{y} \\
\omega_{z}
\end{array}\right]=\left[\begin{array}{c}
-p \sin \Lambda \cos \Gamma-q \cos \Lambda \cos \Gamma-r \sin \Gamma \\
-p \cos \Lambda+q \sin \Lambda \\
p \sin \Lambda \sin \Gamma+q \cos \Lambda \sin \Gamma-r \cos \Gamma
\end{array}\right]}
\end{gathered}
$$

The kinetic energy is formed by

$$
T=\frac{1}{2} \int \rho \mathbf{v} \cdot \mathbf{v} d A=\frac{1}{2} \int \rho\left(v_{x}^{2}+v_{y}^{2}+v_{z}^{2}\right) d A
$$


We use the method of separation of variables to express the displacements as $U(x, t)=\Phi_{u}(x) q_{u}(t)$, $V(x, t)=\Phi_{v}(x) q_{v}(t), W(x, t)=\Phi_{w}(x) q_{w}(t), \Theta(x, t)=\Phi_{\theta}(x) q_{\theta}(t)$. Then, the virtual work quantities due to the generalized coordinates $q_{u}(t), q_{v}(t), q_{w}(t)$, and $q_{\theta}(t)$ are computed in terms of the virtual displacements as

$$
\begin{gathered}
-f_{x}^{i} \delta U=\left[\frac{d}{d t}\left(\frac{\partial T}{\partial \dot{q}_{u}}\right)-\frac{\partial T}{\partial q_{u}}\right] \delta q_{u}=\int \rho\left[\frac{d v_{x}}{d t}-v_{y}\left(\omega_{z}+V_{x t}\right)+v_{z}\left(\omega_{y}-W_{x t}\right)\right] \delta U d A \\
-f_{y}^{i} \delta V=\left[\frac{d}{d t}\left(\frac{\partial T}{\partial \dot{q}_{v}}\right)-\frac{\partial T}{\partial q_{v}}\right] \delta q_{v}=\int \rho\left[\frac{d v_{y}}{d t}+v_{x}\left(\omega_{z}+V_{x t}\right)-v_{z}\left(\omega_{x}+\Theta_{t}\right)\right] \delta V d A \\
+\int \rho\left\{\frac{d\left[v_{x}(-y-V+z \Theta)+v_{y}\left(U-y V_{x}-z W_{x}\right)\right]}{d t}+v_{y}\left(y \omega_{z}+y V_{x t}\right)-v_{z}\left(y \omega_{y}-y W_{x t}\right)\right\} \delta V_{x} d A \\
-f_{z} \delta W=\left[\frac{d}{d t}\left(\frac{\partial T}{\partial \dot{q}_{w}}\right)-\frac{\partial T}{\partial q_{w}}\right] \delta q_{w}=\int \rho\left[\frac{d v_{z}}{d t}-v_{x}\left(\omega_{y}-W_{x t}\right)+v_{y}\left(\omega_{x}+\Theta_{t}\right)\right] \delta W d A \\
+\int \rho\left\{\frac{d\left[v_{x}(-z-W-y \Theta)+v_{z}\left(U-y V_{x}-z W_{x}\right)\right]}{d t}+v_{y}\left(z \omega_{z}+z V_{x t}\right)-v_{z}\left(z \omega_{y}-z W_{x t}\right)\right\} \delta W_{x} d A \\
-m_{x} \delta \Theta=\left[\frac{d}{d t}\left(\frac{\partial T}{\partial \dot{q}_{\theta}}\right)-\frac{\partial T}{\partial q_{\theta}}\right] \delta q_{\theta}=\int \rho\left\{\frac{d\left[v_{y}(-z-W-y \Theta)\right]}{d t}+\frac{d\left[v_{z}(y+V-z \Theta)\right]}{d t}-v_{x}\left(y \omega_{y}+z \omega_{z}+z V_{x t}-y W_{x t}\right)+v_{y}\left(y \omega_{x}+y \Theta_{t}\right)+v_{z}\left(z \omega_{x}+z \Theta_{t}\right)\right\} \delta \Theta d A
\end{gathered}
$$

Let $\int y d A=A e_{c g}$ where $A=\int d A$ is the mass area and $e_{c g}$ is the offset of the CG of a wing section from the elastic axis, positive if the CG lies aft of the elastic axis. We define $I_{x x}=\int\left(y^{2}+z^{2}\right) d A, I_{y y}=\int z^{2} d A$, and $I_{z z}=\int y^{2} d A$. Furthermore, We assume $\int z d A \approx 0$ and $I_{y z}=-\int y z d A \approx 0$. Integrating the integrals that contain $\delta V_{x}$ and $\delta W_{x}$ by parts, we obtain the linear contributions of the aeroelastic deflections to the inertial forces and moment as

$$
\begin{aligned}
& f_{x}^{i}=\rho A\left[-x_{t t}+y_{t} \omega_{z}-z_{t} \omega_{y}+\left(\omega_{y}^{2}+\omega_{z}^{2}\right) U+\left(\dot{\omega}_{z}-\omega_{x} \omega_{y}\right) V-\left(\dot{\omega}_{y}+\omega_{x} \omega_{z}\right) W+2 \omega_{z} V_{t}-2 \omega_{y} W_{t}\right. \\
& \left.+y_{t} V_{x t}+z_{t} W_{x t}-U_{t t}\right]+\rho A e_{c g}\left[-\left(\dot{\omega}_{y}+\omega_{x} \omega_{z}\right) \Theta-\left(\omega_{y}^{2}+\omega_{z}^{2}\right) V_{x}-2 \omega_{y} \Theta_{t}+V_{x t t}\right]+\Delta f_{x}^{i} \\
& f_{y}^{i}=\rho A\left[-y_{t t}-x_{t} \omega_{z}+z_{t} \omega_{x}-\left(\dot{\omega}_{z}+\omega_{x} \omega_{y}\right) U+\left(\omega_{x}^{2}+\omega_{z}^{2}\right) V+\left(\dot{\omega}_{x}-\omega_{y} \omega_{z}\right) W-2 \omega_{z} U_{t}+2 \omega_{x} W_{t}\right. \\
& \left.+z_{t} \Theta_{t}-x_{t} V_{x t}-V_{t t}\right]+\rho A e_{c g}\left[\left(\dot{\omega}_{x}-\omega_{y} \omega_{z}\right) \Theta+\left(\dot{\omega}_{z}+\omega_{x} \omega_{y}\right) V_{x}+2 \omega_{x} \Theta_{t}+2 \omega_{z} V_{x t}\right] \\
& +\frac{\partial}{\partial x}\left[\rho A\left(y_{t t} U-x_{t t} V+y_{t} U_{t}-x_{t} V_{t}\right)\right]+\frac{\partial}{\partial x}\left\{\rho A e _ { c g } \left[-x_{t t}+y_{t} \omega_{z}-z_{t} \omega_{y}+\left(\omega_{y}^{2}+\omega_{z}^{2}\right) U\right.\right. \\
& \left.\left.+\left(\dot{\omega}_{z}-\omega_{x} \omega_{y}\right) V-\left(\dot{\omega}_{y}+\omega_{x} \omega_{z}\right) W-y_{t t} V_{x}+2 \omega_{z} V_{t}-2 \omega_{y} W_{t}+z_{t} W_{x t}-U_{t t}\right]\right\} \\
& +\frac{\partial}{\partial x}\left\{\rho I_{z z}\left[-\left(\dot{\omega}_{y}+\omega_{x} \omega_{z}\right) \Theta-\left(\omega_{y}^{2}+\omega_{z}^{2}\right) V_{x}-2 \omega_{y} \Theta_{t}+V_{x t t}\right]\right\}+\Delta f_{y}^{i} \\
& f_{z}^{i}=\rho A\left[-z_{t t}+x_{t} \omega_{y}-y_{t} \omega_{x}+\left(\dot{\omega}_{y}-\omega_{x} \omega_{z}\right) U-\left(\dot{\omega}_{x}+\omega_{y} \omega_{z}\right) V+\left(\omega_{x}^{2}+\omega_{y}^{2}\right) W+2 \omega_{y} U_{t}-2 \omega_{x} V_{t}\right. \\
& \left.-y_{t} \Theta_{t}-x_{t} W_{x t}-W_{t t}\right]+\rho A e_{c g}\left[\left(\omega_{x}^{2}+\omega_{y}^{2}\right) \Theta-\left(\dot{\omega}_{y}-\omega_{x} \omega_{z}\right) V_{x}-2 \omega_{y} V_{x t}-\Theta_{t t}\right] \\
& +\frac{\partial}{\partial x}\left[\rho A\left(z_{t t} U-x_{t t} W+z_{t} U_{t}-x_{t} W_{t}\right)\right]+\frac{\partial}{\partial x}\left[\rho A e_{c g}\left(-x_{t t} \Theta-z_{t t} V_{x}-x_{t} \Theta_{t}-z_{t} V_{x t}\right)\right] \\
& +\frac{\partial}{\partial x}\left\{\rho I_{y y}\left[-\left(\dot{\omega}_{z}-\omega_{x} \omega_{y}\right) \Theta-\left(\omega_{y}^{2}+\omega_{z}^{2}\right) W_{x}-2 \omega_{z} \Theta_{t}+W_{x t t}\right]\right\}+\Delta f_{z}^{i} \\
& m_{x}^{i}=\rho A\left(-z_{t t} V+y_{t t} W-z_{t} V_{t}+y_{t} W_{t}\right)+\rho A e_{c g}\left[-z_{t t}+x_{t} \omega_{y}-y_{t} \omega_{x}+\left(\dot{\omega}_{y}-\omega_{x} \omega_{z}\right) U\right. \\
& \left.-\left(\dot{\omega}_{x}+\omega_{y} \omega_{z}\right) V+\left(\omega_{x}^{2}+\omega_{y}^{2}\right) W+y_{t t} \Theta+2 \omega_{y} U_{t}-2 \omega_{x} V_{t}-x_{t} W_{x t}-W_{t t}\right]-\rho I_{x x} \Theta_{t t} \\
& +\rho I_{y y}\left[\left(\omega_{x}^{2}+\omega_{z}^{2}\right) \Theta-\left(\dot{\omega}_{z}+\omega_{x} \omega_{y}\right) W_{x}-2 \omega_{z} W_{x t}\right]+\rho I_{z z}\left[\left(\omega_{x}^{2}+\omega_{y}^{2}\right) \Theta-\left(\dot{\omega}_{y}-\omega_{x} \omega_{z}\right) V_{x}\right. \\
& \left.-2 \omega_{y} V_{x t}\right]+\Delta m_{x}^{i}
\end{aligned}
$$


These equations show complex coupling of inertial forces to aeroelasticity. The terms $V_{x t t}$ and $W_{x t t}$ represent the effect of the rotary inertia in bending. The nonlinear contributions are obtained as

$$
\begin{aligned}
& \Delta f_{x}^{i}= \rho A\left(-\omega_{y} V \Theta_{t}-\omega_{z} W \Theta_{t}+2 \omega_{z} U V_{x t}-\omega_{x} W V_{x t}+2 V_{t} V_{x t}-2 \omega_{y} U W_{x t}+\omega_{x} V W_{x t}+2 W_{t} W_{x t}\right. \\
&+\left.V V_{x t t}+W W_{x t t}\right)+\rho A e_{c g}\left(-\omega_{z} \Theta \Theta_{t}-\omega_{x} \Theta V_{x t}-2 \omega_{z} V_{x} V_{x t}+2 \omega_{y} V_{x} W_{x t}+2 \Theta_{t} W_{x t}+\Theta W_{x t t}\right) \\
& \Delta f_{y}^{i}= \rho A\left(-\omega_{y} U \Theta_{t}+2 \omega_{x} V \Theta_{t}+2 W_{t} \Theta_{t}+2 \omega_{z} V V_{x t}-\omega_{y} W V_{x t}-2 U_{t} V_{x t}+\omega_{x} U W_{x t}+\omega_{z} W W_{x t}\right. \\
&\left.+W \Theta_{t t}-U V_{x t t}\right)+\rho A e_{c g}\left(\omega_{y} V_{x} \Theta_{t}+2 \Theta_{t}^{2}-\omega_{y} \Theta V_{x t}+2 V_{x t}^{2}+\omega_{z} \Theta W_{x t}-\omega_{x} V_{x} W_{x t}+\Theta \Theta_{t t}\right. \\
&+\left.V_{x} V_{x t t}\right)+\frac{\partial}{\partial x}\left[\rho A \left(\dot{\omega}_{z} U^{2}+\dot{\omega}_{z} V^{2}-\dot{\omega}_{x} U W-\dot{\omega}_{y} V W+2 \omega_{z} U U_{t}-\omega_{x} W U_{t}+2 \omega_{z} V V_{t}-\omega_{y} W V_{t}\right.\right. \\
&\left.\left.-\omega_{x} U W_{t}-\omega_{y} V W_{t}-V U_{t t}+U V_{t t}\right)\right]+\frac{\partial}{\partial x}\left[\rho A e _ { c g } \left(-\dot{\omega}_{x} U \Theta-\dot{\omega}_{y} V \Theta-2 \dot{\omega}_{z} U V_{x}+\dot{\omega}_{x} W V_{x}\right.\right. \\
&- \omega_{x} \Theta U_{t}-2 \omega_{z} V_{x} U_{t}-\omega_{y} \Theta V_{t}+\omega_{x} V_{x} W_{t}-\omega_{x} U \Theta_{t}-2 \omega_{y} V \Theta_{t}-\omega_{z} W \Theta_{t}+2 V_{t} V_{x t}-2 \omega_{y} U W_{x t} \\
&\left.\left.+\omega_{x} V W_{x t}+2 W_{t} W_{x t}-V_{x} V_{t t}+2 V V_{x t t}+W W_{x t t}\right)\right]+\frac{\partial}{\partial x}\left[\rho I _ { y y } \left(\dot{\omega}_{z} \Theta^{2}+\dot{\omega}_{z} W_{x}^{2}+2 \omega_{z} \Theta \Theta_{t}\right.\right. \\
&+\left.\left.2 \omega_{z} W_{x} W_{x t}+W_{x} \Theta_{t t}-\Theta W_{x t t}\right)\right]+\frac{\partial}{\partial x}\left[\rho I _ { z z } \left(\dot{\omega}_{x} \Theta V_{x}+\dot{\omega}_{z} V_{x}^{2}-\omega_{z} \Theta \Theta_{t}+\omega_{x} V_{x} \Theta_{t}+2 \omega_{y} V_{x} W_{x t}\right.\right. \\
&\left.\left.+2 \Theta_{t} W_{x t}+\Theta W_{x t t}\right)\right]
\end{aligned}
$$

$$
\begin{array}{r}
\Delta f_{z}^{i}=\rho A\left(-\omega_{z} U \Theta_{t}+2 \omega_{x} W \Theta_{t}+y \omega_{z} V_{x} \Theta_{t}-2 V_{t} \Theta_{t}-\omega_{x} U V_{x t}-\omega_{y} V V_{x t}+\omega_{z} V W_{x t}-2 \omega_{y} W W_{x t}\right. \\
\left.-2 U_{t} W_{x t}-V \Theta_{t t}-U W_{x t t}\right)+\rho A e_{c g}\left(2 \omega_{x} \Theta \Theta_{t}+\omega_{x} V_{x} V_{x t}-2 \omega_{y} \Theta W_{x t}+2 V_{x t} W_{x t}+V_{x} W_{x t t}\right) \\
+\frac{\partial}{\partial x}\left[\rho A \left(-\dot{\omega}_{y} U^{2}+\dot{\omega}_{x} U V+\dot{\omega}_{z} V W-\dot{\omega}_{y} W^{2}-2 \omega_{y} U U_{t}+\omega_{x} V U_{t}+\omega_{x} U V_{t}+\omega_{z} W V_{t}\right.\right. \\
\left.\left.+\omega_{z} V W_{t}-2 \omega_{y} W W_{t}-W U_{t t}+U W_{t t}\right)\right]+\frac{\partial}{\partial x}\left[\rho A e _ { c g } \left(\dot{\omega}_{z} V \Theta-2 \dot{\omega}_{y} W \Theta+2 \dot{\omega}_{y} U V_{x}-\dot{\omega}_{x} V V_{x}\right.\right. \\
+2 \omega_{y} V_{x} U_{t}-\omega_{x} V_{x} V_{t}-2 \omega_{y} \Theta W_{t}+2 \omega_{z} V \Theta_{t}-2 \omega_{y} W \Theta_{t}+2 \omega_{y} U V_{x t}-\omega_{x} V V_{x t}-\Theta U_{t t}-V_{x} W_{t t} \\
\left.\left.+U \Theta_{t t}+W V_{x t t}\right)\right]+\frac{\partial}{\partial x}\left[\rho I _ { y y } \left(\dot{\omega}_{x} \Theta W_{x}-\dot{\omega}_{y} W_{x}^{2}+\omega_{y} \Theta \Theta_{t}+\omega_{x} W_{x} \Theta_{t}-2 \omega_{z} W_{x} V_{x t}-2 \Theta_{t} V_{x t}\right.\right. \\
\left.\left.-\Theta V_{x t t}\right)\right]+\frac{\partial}{\partial x}\left[\rho I_{z z}\left(-\dot{\omega}_{y} \Theta^{2}-\dot{\omega}_{y} V_{x}^{2}-2 \omega_{y} \Theta \Theta_{t}-2 \omega_{y} V_{x} V_{x t}-V_{x} \Theta_{t t}+\Theta V_{x t t}\right)\right]
\end{array}
$$

$$
\begin{gathered}
\Delta m_{x}^{i}=\rho A\left(\dot{\omega}_{y} U V-\dot{\omega}_{x} V^{2}+\dot{\omega}_{z} U W-\dot{\omega}_{x} W^{2}+\omega_{y} V U_{t}+\omega_{z} W U_{t}+\omega_{y} U V_{t}-2 \omega_{x} V V_{t}+\omega_{z} U W_{t}\right. \\
\left.-2 \omega_{x} W W_{t}+W V_{t t}-V W_{t t}\right)+\rho A e_{c g}\left(\dot{\omega}_{z} U \Theta-2 \dot{\omega}_{x} W \Theta-\dot{\omega}_{y} V V_{x}-\dot{\omega}_{z} W V_{x}+\omega_{z} \Theta U_{t}-\omega_{y} V_{x} V_{t}\right. \\
-2 \omega_{x} \Theta W_{t}-\omega_{z} V_{x} W_{t}-\omega_{x} U V_{x t}-2 V_{t} \Theta_{t}-2 \omega_{y} V V_{x t}-\omega_{z} W V_{x t}+\omega_{z} V W_{x t}-2 \omega_{y} W W_{x t}-2 U_{t} W_{x t} \\
\left.\quad+\Theta V_{t t}-2 V \Theta_{t t}-U W_{x t t}\right)-\rho I_{x x} \dot{\omega}_{x} \Theta+\rho I_{y y}\left(\dot{\omega}_{y} \Theta W_{x}+2 \omega_{z} \Theta V_{x t}+\omega_{y} \Theta W_{x t}+\omega_{x} W_{x} W_{x t}\right. \\
\left.-2 V_{x t} W_{x t}-W_{x} V_{x t t}\right)+\rho I_{z z}\left(-\dot{\omega}_{z} \Theta V_{x}-\omega_{z} \Theta V_{x t}+\omega_{x} V_{x} V_{x t}-2 \omega_{y} \Theta W_{x t}+2 V_{x t} W_{x t}+V_{x} W_{x t t}\right)
\end{gathered}
$$

\section{Strain Analysis}

Differentiating $x_{1}, y_{1}$, and $z_{1}$ with respect to $x$ yields

$$
\left[\begin{array}{c}
x_{1, x} \\
y_{1, x} \\
z_{1, x}
\end{array}\right]=\left[\begin{array}{c}
1+U_{x}-y V_{x x}+z \gamma^{\prime} V_{x}-z W_{x x}-y \gamma^{\prime} W_{x} \\
-z \gamma^{\prime}+V_{x}-z \Theta_{x}-y \gamma^{\prime} \Theta \\
y \gamma^{\prime}+W_{x}+y \Theta_{x}-z \gamma^{\prime} \Theta
\end{array}\right]
$$

Neglecting the transverse shear effect, the longitudinal strain is computed as ${ }^{8}$

$$
\epsilon=\frac{d s_{1}-d s}{d s}=\frac{s_{1, x}}{s_{x}}-1
$$


where

$$
s_{x}=\sqrt{1+y_{x}^{2}+z_{x}^{2}}=\sqrt{1+\left(y^{2}+z^{2}\right)\left(\gamma^{\prime}\right)^{2}}
$$

$$
\begin{aligned}
s_{1, x} & =\sqrt{x_{1, x}^{2}+y_{1, x}^{2}+z_{1, x}^{2}} \\
& =\sqrt{s_{x}^{2}+2 U_{x}-2 y V_{x x}-2 z W_{x x}+2\left(y^{2}+z^{2}\right) \gamma^{\prime} \Theta_{x}+\left(x_{1, x}-1\right)^{2}+\left(y_{1, x}+z \gamma^{\prime}\right)^{2}+\left(z_{1, x}-y \gamma^{\prime}\right)^{2}}
\end{aligned}
$$

$s_{1, x}$ is approximated by a Taylor series as

$$
s_{1, x} \approx s_{x}+\frac{U_{x}-y V_{x x}-z W_{x x}+\left(y^{2}+z^{2}\right) \gamma^{\prime} \Theta_{x}}{s_{x}}+\frac{\left(x_{1, x}-1\right)^{2}+\left(y_{1, x}+z \gamma^{\prime}\right)^{2}+\left(z_{1, x}-y \gamma^{\prime}\right)^{2}}{2 s_{x}}+\cdots
$$

The slope of the twist angle $\gamma^{\prime}$ can play a significant role in structures with large twists such as turbomachinery blades. For aircraft wings, this effect is negligible and therefore can be neglected. Thus, for a small wing twist angle $\gamma, \gamma^{\prime} \approx 0$ and $s_{x} \approx 1$. The longitudinal strain is then obtained as

$$
\begin{aligned}
\epsilon=U_{x}-y V_{x x}-z W_{x x}+\frac{1}{2} U_{x}^{2}+\frac{1}{2} V_{x}^{2}+\frac{1}{2} W_{x}^{2} & +\frac{1}{2}\left(y^{2}+z^{2}\right) \Theta_{x}^{2}+\frac{1}{2} y^{2} V_{x x}^{2}+\frac{1}{2} z^{2} W_{x x}^{2} \\
& -y U_{x} V_{x x}-z U_{x} W_{x x}+y z V_{x x} W_{x x}-z V_{x} \Theta_{x}+y W_{x} \Theta_{x}
\end{aligned}
$$

The slope of the twist angle $\gamma^{\prime}$ can play a significant role in structures with large twists such as turbomachinery blades. For aircraft wings, this effect is negligible and therefore can be neglected. Assuming $\bar{z} \approx 0$ and letting $\bar{y}=e_{a}$ where $e_{a}$ is the offset of the neutral axis on a wing section from the elastic axis, positive if the centroid lies aft of the elastic axis, then the axial force and moments acting on a wing are evaluated $\operatorname{as}^{8}$

$$
\begin{gathered}
P_{x}=\int E \epsilon d A=E A U_{x}-E A e_{a} V_{x x}+\frac{1}{2} E A\left(U_{x}^{2}+V_{x}^{2}+W_{x}^{2}\right)+\frac{1}{2} E I_{x x} \Theta_{x}^{2}+E A e_{a} W_{x} \Theta_{x} \\
+\frac{1}{2} E I_{z z} V_{x x}^{2}+\frac{1}{2} E I_{y y} W_{x x}^{2}-\left(E A e_{a} U_{x}+E I_{y z} W_{x x}\right) V_{x x} \\
M_{x}=G J \Theta_{x}+\int E \epsilon\left(y^{2}+z^{2}\right) \Theta_{x} d A=\left[G J+E I_{x x} U_{x}-E B_{3} V_{x x}-E B_{2} W_{x x}\right. \\
+\frac{1}{2} E I_{x x}\left(U_{x}^{2}+V_{x}^{2}+W_{x}^{2}\right)+\frac{1}{2} E B_{4} V_{x x}^{2}+\frac{1}{2} E B_{5} W_{x x}^{2}+E B_{6} V_{x x} W_{x x} \\
\left.-\left(E B_{3} V_{x x}+E B_{2} W_{x x}\right) U_{x}\right] \Theta_{x}+\left(-E B_{2} V_{x}+E B_{3} W_{x}\right) \Theta_{x}^{2}+\frac{1}{2} E B_{1} \Theta_{x}^{3} \\
M_{y}=-\int E \epsilon z d A=\left(1+U_{x}\right)\left(E I_{y y} W_{x x}-E I_{y z} V_{x x}\right)-\frac{1}{2} E B_{2} \Theta_{x}^{2}-\frac{1}{2} E B_{7} V_{x x}^{2}-\frac{1}{2} E B_{8} W_{x x}^{2} \\
-E B_{9} V_{x x} W_{x x}+\left(E I_{y y} V_{x}+E I_{y z} W_{x}\right) \Theta_{x} \\
M_{z}=-\int E \epsilon y d A=-E A e_{a} U_{x}+\left(1+U_{x}\right)\left(-E I_{y z} W_{x x}+E I_{z z} V_{x x}\right)-\frac{1}{2} E A e_{a}\left(U_{x}^{2}+V_{x}^{2}+W_{x}^{2}\right) \\
-\frac{1}{2} E B_{3} \Theta_{x}^{2}-\frac{1}{2} E B_{10} V_{x x}^{2}-\frac{1}{2} E B_{9} W_{x x}^{2}-E B_{7} V_{x x} W_{x x}-\left(E I_{y z} V_{x}+E I_{z z} W_{x}\right) \Theta_{x}
\end{gathered}
$$

where $E$ is the Young's modulus, $G$ is the shear modulus, $A$ is the tensile area, $J$ is the torsional constant, 
and $B_{i}, i=1,2, \ldots, 10$ are defined as

$$
\left[\begin{array}{c}
B_{1} \\
B_{2} \\
B_{3} \\
B_{4} \\
B_{5} \\
B_{6} \\
B_{7} \\
B_{8} \\
B_{9} \\
B_{10}
\end{array}\right]=\int\left[\begin{array}{c}
\left(y^{2}+z^{2}\right)^{2} \\
z\left(y^{2}+z^{2}\right) \\
y\left(y^{2}+z^{2}\right) \\
y^{2}\left(y^{2}+z^{2}\right) \\
z^{2}\left(y^{2}+z^{2}\right) \\
y z\left(y^{2}+z^{2}\right) \\
y^{2} z \\
z^{3} \\
y z^{2} \\
y^{3}
\end{array}\right] d A
$$

Note that the signs of the moments are defined in the positive deflection sense such that

$$
\mathbf{M}=M_{x} \mathbf{d}_{1}-M_{y} \mathbf{d}_{2}+M_{z} \mathbf{d}_{3}
$$

For truss-braced wing aircraft configurations, the effect of the axial displacement can be significant and therefore must be included in the strain analysis. Assuming the chordwise bending deflection is small and neglecting cross-product inertia $I_{y z}, B_{2}, B_{6}, B_{7}, B_{8}$ which are generally smaller than the remaining $B_{i}$, then the following simplification can be made:

$$
\begin{gathered}
P_{x}=E A U_{x}-E A e_{a} V_{x x}+\frac{1}{2} E A\left(U_{x}^{2}+W_{x}^{2}\right)+\frac{1}{2} E I_{x x} \Theta_{x}^{2}+E A e_{a} W_{x} \Theta_{x}+\frac{1}{2} E I_{y y} W_{x x}^{2} \\
M_{x}=\left[G J+E I_{x x} U_{x}+\frac{1}{2} E I_{x x}\left(U_{x}^{2}+W_{x}^{2}\right)+\frac{1}{2} E B_{5} W_{x x}^{2}\right] \Theta_{x}+E B_{3} W_{x} \Theta_{x}^{2}+\frac{1}{2} E B_{1} \Theta_{x}^{3} \\
M_{y}=\left(1+U_{x}\right) E I_{y y} W_{x x} \\
M_{z}=-E A e_{a} U_{x}+\left(1+U_{x}\right) E I_{z z} V_{x x}-\frac{1}{2} E A e_{a}\left(U_{x}^{2}+W_{x}^{2}\right)-\frac{1}{2} E B_{3} \Theta_{x}^{2}-\frac{1}{2} E B W_{x x}^{2}-E I_{z z} W_{x} \Theta_{x}
\end{gathered}
$$

\section{Aeroelastic Analysis}

In order to compute the aeroelastic forces and moments, the velocity must be transformed from the reference frame $\mathrm{D}$ to the airfoil local coordinate reference frame defined by $(\mu, \eta, \xi)$ as follows:

$$
\left[\begin{array}{l}
v_{\mu} \\
v_{\eta} \\
v_{\xi}
\end{array}\right]=\left[\begin{array}{ccc}
1 & 0 & 0 \\
0 & \cos (\Theta+\gamma) & \sin (\Theta+\gamma) \\
0 & -\sin (\Theta+\gamma) & \cos (\Theta+\gamma)
\end{array}\right]\left[\begin{array}{ccc}
\cos V_{x} & \sin V_{x} & 0 \\
-\sin V_{x} & \cos V_{x} & 0 \\
0 & 0 & 1
\end{array}\right]\left[\begin{array}{ccc}
\cos W_{x} & 0 & \sin W_{x} \\
0 & 1 & 0 \\
-\sin W_{x} & 0 & \cos W_{x}
\end{array}\right]\left[\begin{array}{c}
v_{x} \\
v_{y} \\
v_{z}
\end{array}\right]
$$

Neglecting higher-order terms and assuming $\gamma \approx 0$, then the velocity components in the local coordinate reference frame are computed as

$$
\left[\begin{array}{c}
v_{\mu} \\
v_{\eta} \\
v_{\xi}
\end{array}\right] \approx\left[\begin{array}{c}
v_{x}+v_{y} V_{x}+v_{z} W_{x} \\
-v_{x}\left(V_{x}+W_{x} \Theta\right)+v_{y}+v_{z}\left[\Theta-V_{x} W_{x}\right] \\
v_{x}\left(-W_{x}+V_{x} \Theta\right)-v_{y} \Theta+v_{z}
\end{array}\right]
$$

The local aeroelastic angle of attack on the airfoil section due to the velocity components $v_{\eta}$ and $v_{\xi}$ in the reference frame D, as shown in Fig. 8, is computed as

$$
\alpha_{c}=\frac{v_{\xi}}{v_{\eta}}=\frac{\bar{v}_{\xi}+\Delta v_{\xi}}{\bar{v}_{\eta}+\Delta v_{\eta}}=\frac{v_{\xi}}{\bar{v}_{\eta}}-\frac{\bar{v}_{\xi} \Delta v_{\eta}}{\bar{v}_{\eta}^{2}}
$$

where

$$
\bar{v}_{\xi}=z_{t}
$$




$$
\begin{gathered}
\Delta v_{\xi}=W_{t}+\left(U-y V_{x}-z W_{x}\right)\left(W_{x t}-\omega_{y}\right)+(y+V-z \Theta) \Theta_{t}+\omega_{x}(V-z \Theta)+x_{t}\left(-W_{x}+V_{x} \Theta\right) \\
-\left[-\omega_{z}(V-z \Theta)+\omega_{y}(W+y \Theta)+U_{t}-y V_{x t}-z W_{x t}\right] W_{x} \\
-\left[y_{t}+\omega_{z}\left(U-y V_{x}-z W_{x}\right)-\omega_{x}(W+y \Theta)+V_{t}-z \Theta_{t}\right] \Theta+z_{t} \\
\bar{v}_{\eta}=-u \cos \Lambda
\end{gathered}
$$

$$
\begin{aligned}
\Delta v_{\eta}=V_{t}+\left(U-y V_{x}-z W_{x}\right)\left(V_{x t}+\omega_{z}\right)- & (z+W+y \Theta) \Theta_{t}-\omega_{x}(W+y \Theta)-x_{t}\left(V_{x}+W_{x} \Theta\right) \\
-\left[-\omega_{z}(V-z \Theta)+\omega_{y}(W+y \Theta)\right. & \left.+U_{t}-y V_{x t}-z W_{x t}\right] V_{x}+y_{t}-\bar{v}_{\eta}+z_{t}\left(\Theta-V_{x} W_{x}\right) \\
& +\left[-\omega_{y}\left(U-y V_{x}-z W_{x}\right)+\omega_{x}(V-z \Theta)+W_{t}+y \Theta_{t}\right] \Theta
\end{aligned}
$$

\begin{tabular}{|c|c|c|c|c|c|c|c|}
\hline & 1 & $U$ & $V$ & $W$ & $\Theta$ & $V_{x}$ & $W_{x}$ \\
\hline 1 & & $\begin{aligned} & \frac{\omega_{y}}{u \cos \Lambda} \\
&- \frac{z_{t} \omega_{z}}{u^{2} \cos ^{2} \Lambda} \\
&\end{aligned}$ & $-\frac{\omega_{x}}{u \cos \Lambda}$ & $\frac{z_{t} \omega_{x}}{u^{2} \cos ^{2} \Lambda}$ & $\begin{array}{c}\frac{y_{t}}{u \cos \Lambda} \\
-\frac{z_{t}\left(-\omega_{x} y+z_{t}\right)}{u^{2} \cos ^{2} \Lambda}\end{array}$ & $\begin{array}{c}-\frac{\omega_{y} y}{u \cos \Lambda} \\
+\frac{z_{t}\left(\omega_{z} y+x_{t}\right)}{u^{2} \cos ^{2} \Lambda}\end{array}$ & $\frac{x_{t}}{u \cos \Lambda}$ \\
\hline$U$ & $\begin{aligned} & \frac{\omega_{y}}{u \cos \Lambda} \\
&- \frac{z+\omega_{z}}{u^{2} \cos ^{2} \Lambda} \\
&\end{aligned}$ & 0 & 0 & 0 & $\begin{array}{c}\quad \frac{\omega_{z}}{u \cos \Lambda} \\
+\frac{z_{t} \omega_{y}}{u \cos ^{2} \Lambda} \\
\end{array}$ & $\begin{array}{c}u^{2} \cos ^{2} \Lambda \\
0\end{array}$ & 0 \\
\hline V & $-\frac{\omega_{x}}{u \cos \Lambda}$ & 0 & 0 & 0 & $-\frac{z_{t} \omega_{x}}{u^{2} \cos \Lambda}$ & $-\frac{z_{t} \omega_{z}}{u^{2} \cos \Lambda}$ & $\begin{array}{c}-\frac{\omega_{z}}{u \cos \Lambda} \\
+\frac{z_{t}^{2}}{u^{2} \cos ^{2} \Lambda}\end{array}$ \\
\hline$W$ & $\frac{z_{t} \omega_{x}}{u^{2} \cos ^{2} \Lambda}$ & 0 & 0 & 0 & $-\frac{\omega_{x}}{u \cos \Lambda}$ & $\frac{z_{t} \omega_{y}}{u^{2} \cos \Lambda}$ & $\frac{\omega_{y}}{u \cos \Lambda}$ \\
\hline$\Theta$ & $\begin{array}{c}\frac{y_{t}}{u \cos \Lambda} \\
-\frac{z_{t}\left(-\omega_{x} y+z_{t}\right)}{u^{2} \cos ^{2} \Lambda} \\
\end{array}$ & $\begin{array}{c}\frac{\omega_{z}}{u \cos \Lambda} \\
+\frac{z_{t} \omega_{y}}{u \cos ^{2} \Lambda}\end{array}$ & $-\frac{z_{t} \omega_{x}}{u^{2} \cos \Lambda}$ & $-\frac{\omega_{x}}{u \cos \Lambda}$ & $-\frac{\omega_{x} y}{u \cos \Lambda}$ & $\begin{array}{l}-\frac{x_{t}+\omega_{z} y}{u \cos \Lambda} \\
-\frac{z_{t} \omega_{y} y}{u^{2} \cos ^{2} \Lambda}\end{array}$ & $\frac{z_{t} x_{t}}{u^{2} \cos \Lambda}$ \\
\hline$V_{x}$ & $\begin{array}{c}-\frac{\omega_{y} y}{u \cos \Lambda} \\
+\frac{z_{t}\left(\omega_{z} y+x_{t}\right)}{u^{2} \cos ^{2} \Lambda} \\
\end{array}$ & 0 & $-\frac{z_{t} \omega_{z}}{u^{2} \cos \Lambda}$ & $\frac{z_{t} \omega_{y}}{u^{2} \cos \Lambda}$ & $\begin{array}{r}-\frac{x_{t}+\omega_{z} y}{u \cos \Lambda} \\
-\frac{z_{t} \omega_{y} y}{u^{2} \cos ^{2} \Lambda}\end{array}$ & 0 & $\frac{z_{t}^{2}}{u^{2} \cos ^{2} \Lambda}$ \\
\hline$W_{x}$ & $\frac{x_{t}}{u \cos \Lambda}$ & 0 & $\begin{array}{c}-\frac{\omega_{z}}{u \cos \Lambda} \\
+\frac{z_{t}^{2}}{u^{2} \cos ^{2} \Lambda}\end{array}$ & $\frac{\omega_{y}}{u \cos \Lambda}$ & $\frac{z_{t} x_{t}}{u^{2} \cos \Lambda}$ & $\frac{z_{t}^{2}}{u^{2} \cos ^{2} \Lambda}$ & 0 \\
\hline$U_{t}$ & 0 & 0 & 0 & 0 & 0 & $\frac{z_{t}}{u^{2} \cos ^{2} \Lambda}$ & $\frac{1}{u \cos \Lambda}$ \\
\hline$\overline{V_{t}}$ & $-\frac{z_{t}}{u^{2} \cos ^{2} \Lambda}$ & 0 & 0 & 0 & $\frac{1}{u \cos \Lambda}$ & 0 & 0 \\
\hline$W_{t}$ & $-\frac{1}{u \cos \Lambda}$ & 0 & 0 & 0 & $-\frac{z_{t}}{u^{2} \cos ^{2} \Lambda}$ & 0 & 0 \\
\hline$\Theta_{t}$ & $-\frac{y}{u \cos \Lambda}$ & 0 & $-\frac{1}{u \cos \Lambda}$ & $\frac{z_{t}}{u^{2} \cos ^{2} \Lambda}$ & 0 & 0 & 0 \\
\hline$V_{x t}$ & 0 & 0 & 0 & 0 & 0 & $-\frac{z_{t} y}{u^{2} \cos ^{2} \Lambda}$ & $-\frac{y}{u \cos \Lambda}$ \\
\hline$W_{x t}$ & 0 & $-\frac{1}{u \cos \Lambda}$ & 0 & 0 & 0 & 0 & 0 \\
\hline
\end{tabular}

The rigid-body angle of attack is computed as

$$
\alpha_{r}(x)=-\frac{z_{t}}{u \cos \Lambda}\left(1+\frac{y_{t}+u \cos \Lambda}{u \cos \Lambda}\right)
$$

Assuming $z \approx 0$, then the partial derivatives of the local aeroelastic angle of attack are evaluated as shown in Table 1.

Table 1. Partial Derivatives of Aeroelastic Angle of Attack

The aeroelastic angle of attack can be expressed as

$$
\begin{gathered}
\alpha_{e}(x, y)=\frac{\partial \alpha_{e}}{\partial U} U+\frac{\partial \alpha_{e}}{\partial V} V+\frac{\partial \alpha_{e}}{\partial W} W+\frac{\partial \alpha_{e}}{\partial \Theta} \Theta+\frac{\partial \alpha_{e}}{\partial V_{x}} V_{x}+\frac{\partial \alpha_{e}}{\partial W_{x}} W_{x}+\frac{\partial \alpha_{e}}{\partial V_{t}} V_{t}+\frac{\partial \alpha_{e}}{\partial W_{t}} W_{t}+\frac{\partial \alpha_{e}}{\partial \Theta_{t}} \Theta_{t} \\
+\frac{\partial \alpha_{e}}{\partial(U \Theta)} U \Theta+\frac{\partial \alpha_{e}}{\partial(V \Theta)} V \Theta+\frac{\partial \alpha_{e}}{\partial\left(V V_{x}\right)} V V_{x}+\frac{\partial \alpha_{e}}{\partial\left(V W_{x}\right)} V W_{x}+\frac{\partial \alpha_{e}}{\partial(W \Theta)} W \Theta+\frac{\partial \alpha_{e}}{\partial\left(W V_{x}\right)} W V_{x} \\
+\frac{\partial \alpha_{e}}{\partial\left(W W_{x}\right)} W W_{x}+\frac{\partial \alpha_{e}}{\partial\left(\Theta^{2}\right)} \Theta^{2}+\frac{\partial \alpha_{e}}{\partial\left(\Theta V_{x}\right)} \Theta V_{x}+\frac{\partial \alpha_{e}}{\partial\left(\Theta W_{x}\right)} \Theta W_{x}+\frac{\partial \alpha_{e}}{\partial\left(V_{x} W_{x}\right)} V_{x} W_{x}+\frac{\partial \alpha_{e}}{\partial\left(U_{t} V_{x}\right)} U_{t} V_{x} \\
+\frac{\partial \alpha_{e}}{\partial\left(U_{t} W_{x}\right)} U_{t} W_{x}+\frac{\partial \alpha_{e}}{\partial\left(V_{t} \Theta\right)} V_{t} \Theta+\frac{\partial \alpha_{e}}{\partial\left(W_{t} \Theta\right)} W_{t} \Theta+\frac{\partial \alpha_{e}}{\partial\left(V_{x t} V_{x}\right)} V_{x t} V_{x}+\frac{\partial \alpha_{e}}{\partial\left(V_{x t} W_{x}\right)} V_{x t} W_{x} \\
+\frac{\partial \alpha_{e}}{\partial\left(W_{x t} U\right)} W_{x t} U
\end{gathered}
$$


The partial derivatives can be read from Table 1, e.g., $\frac{\partial \alpha_{c}}{\partial U}=\frac{x_{t}}{u \cos \Lambda}$ and $\frac{\partial \alpha_{c}}{\partial\left(\Theta^{2}\right)}=-\frac{\omega_{x} y}{u \cos \Lambda}$. Note that the inertial coupling gives rise to additional stiffness terms $\frac{\partial \alpha_{c}}{\partial U}, \frac{\partial \alpha_{c}}{\partial V}$, and $\frac{\partial \alpha_{c}}{\partial W}$ for linear aeroelasticity.

If chordwise bending is small and therefore can be neglected, then

$$
\begin{array}{r}
\alpha_{e}(x, y)=\frac{\partial \alpha_{e}}{\partial U} U+\frac{\partial \alpha_{e}}{\partial V} V+\frac{\partial \alpha_{e}}{\partial W} W+\frac{\partial \alpha_{e}}{\partial \Theta} \Theta+\frac{\partial \alpha_{e}}{\partial V_{x}} V_{x}+\frac{\partial \alpha_{e}}{\partial W_{x}} W_{x}+\frac{\partial \alpha_{e}}{\partial V_{t}} V_{t}+\frac{\partial \alpha_{e}}{\partial W_{t}} W_{t}+\frac{\partial \alpha_{e}}{\partial \Theta_{t}} \Theta_{t} \\
+\frac{\partial \alpha_{e}}{\partial(U \Theta)} U \Theta+\frac{\partial \alpha_{e}}{\partial(W \Theta)} W \Theta+\frac{\partial \alpha_{e}}{\partial\left(W W_{x}\right)} W W_{x}+\frac{\partial \alpha_{e}}{\partial\left(\Theta^{2}\right)} \Theta^{2}+\frac{\partial \alpha_{e}}{\partial\left(\Theta W_{x}\right)} \Theta W_{x}+\frac{\partial \alpha_{e}}{\partial\left(U_{t} W_{x}\right)} U_{t} W_{x} \\
+\frac{\partial \alpha_{e}}{\partial\left(W_{t} \Theta\right)} W_{t} \Theta+\frac{\partial \alpha_{e}}{\partial\left(W_{x t} U\right)} W_{x t} U
\end{array}
$$

For circulatory lift, the aeroelastic angle of attack is evaluated by setting $y=e_{c}$ where $e_{c}$ is the offset of the unsteady aerodynamic center at the three-quarter point from the elastic center. The circulatory lift and pitching moment coefficients are given by

$$
\begin{gathered}
c_{L_{c}}=\left[c_{L_{\alpha}} \alpha_{r}+C(k) c_{L_{\alpha}} \alpha_{e}\left(x, e_{c}\right)\right] \cos \Lambda \\
c_{m_{c}}=c_{m_{a c}}+\frac{e}{c}\left[c_{L_{\alpha}} \alpha_{r}+C(k) c_{L_{\alpha}} \alpha_{e}\left(x, e_{c}\right)\right] \cos \Lambda
\end{gathered}
$$

where $C(k)$ is the Theodorsen function, ${ }^{10} c_{L_{\alpha}}$ is the lift curve slope, $c_{m_{a c}}$ is the pitching moment coefficient about the aerodynamic center, $c$ is the chord length in the streamwise direction, $e$ is the offset of the aerodynamic center from the elastic axis in the streamwise direction.

For the non-circulatory lift, we express the aeroelastic angle of attack as

$$
\alpha_{e}(x, y)=\alpha_{1}(x)+\alpha_{2}(x, \bar{y})
$$

where $y=\bar{y} \cos \Lambda$ and $\bar{y}$ is the airfoil coordinate along the streamwise direction.

Then the velocity potential according to Theodorsen ${ }^{11}$ is given by

$$
\phi=\frac{V_{\infty} \cos \Lambda c}{2}\left[\alpha_{1}+\frac{\alpha_{2}+\alpha_{2}\left(x, e_{m}\right)}{2}\right] \sqrt{1-\xi^{2}}
$$

where $\bar{y}=\frac{c}{2} \xi+e_{m}$.

The non-circulatory lift is evaluated as

$$
\begin{array}{r}
l_{n c}=\rho_{\infty} c \int_{-1}^{1} \frac{\partial \phi}{\partial t} d \xi=\frac{\rho_{\infty} V_{\infty} \cos \Lambda c^{2}}{2} \int_{-1}^{1}\left[\frac{\partial \alpha_{1}}{\partial t}+\frac{1}{2} \frac{\partial \alpha_{2}}{\partial t}+\frac{1}{2} \frac{\partial \alpha_{2}\left(x, e_{m}\right)}{\partial t}\right] \sqrt{1-\xi^{2}} d \xi \\
=\frac{\pi \rho_{\infty} V_{\infty} \cos \Lambda c^{2}}{4} \frac{\partial \alpha_{e}\left(x, e_{m}\right)}{\partial t}
\end{array}
$$

which yields the non-circulatory lift coefficient as

$$
c_{L_{n c}}=\frac{2 l_{n c}}{\rho_{\infty} V_{\infty}^{2} c}=\frac{\pi \cos \Lambda c}{2 V_{\infty}} \frac{\partial \alpha_{e}\left(x, e_{m}\right)}{\partial t}
$$

The non-circulatory pitching moment is evaluated as

$$
m_{n c}=-\frac{\pi \alpha_{e}\left(x, e_{c}\right) q_{\infty} c^{2} \cos \Lambda}{2}+\rho_{\infty} V_{\infty} c \int_{-1}^{1} \phi d \xi-\rho c \int_{-1}^{1} y \frac{\partial \phi}{\partial t} d \xi
$$

The resulting expression for the non-circulatory pitching moment is obtained as

$$
\begin{gathered}
m_{n c}=-\frac{2 \pi \alpha_{e}\left(x, e_{c}\right) q_{\infty} c^{2} \cos \Lambda}{4}+\frac{2 \pi \alpha_{e}\left(x, e_{m}\right) q_{\infty} c^{2} \cos \Lambda}{4}-\frac{2 \pi q_{\infty} c^{4} \cos \Lambda}{128 V_{\infty}} \frac{\partial \alpha_{2}(x, 1)}{\partial t} \\
-\frac{2 \pi q_{\infty} c^{2} e_{m} \cos \Lambda}{4 V_{\infty}} \frac{\partial \alpha_{e}\left(x, e_{m}\right)}{\partial t}
\end{gathered}
$$


Further simplification yields

$$
c_{m_{n c}}=\frac{m_{n c}}{q_{\infty} c^{2}}=2 \pi\left[-\frac{\alpha_{e}\left(x, e_{c}\right)}{4}+\frac{\alpha_{e}\left(x, e_{m}\right)}{4}-\frac{c^{2}}{128 V_{\infty}} \frac{\partial \alpha_{2}(x, 1)}{\partial t}-\frac{e_{m}}{4 V_{\infty}} \frac{\partial \alpha_{e}\left(x, e_{m}\right)}{\partial t}\right] \cos \Lambda
$$

Note that

$$
-\frac{\alpha_{e}\left(x, e_{c}\right)}{4}+\frac{\alpha_{e}\left(x, e_{m}\right)}{4}=-\frac{\alpha_{2}\left(x, e_{c}\right)}{4}+\frac{\alpha_{2}\left(x, e_{m}\right)}{4}=-\frac{\alpha_{2}(x, c)}{16}
$$

since $e_{c}-e_{m}=\frac{c}{4}$.

Therefore, the non-circulatory pitching moment coefficient can also be expressed as

$$
c_{m_{n c}}=\frac{m_{n c}}{q_{\infty} c^{2}}=-2 \pi \cos \Lambda\left[\frac{\alpha_{2}(x, c)}{16}+\frac{c^{2}}{128 V_{\infty}} \frac{\partial \alpha_{2}(x, 1)}{\partial t}+\frac{e_{m}}{4 V_{\infty}} \frac{\partial \alpha_{e}\left(x, e_{m}\right)}{\partial t}\right]
$$

The total lift and pitching moment coefficients are

$$
\begin{aligned}
c_{L} & =c_{L_{c}}+c_{L_{n c}} \\
c_{m} & =c_{m_{c}}+c_{m_{n c}}
\end{aligned}
$$

The total aerodynamic forces and moments are due to both circulatory lift and non-circulatory lift

$$
\begin{gathered}
f_{x}^{a}=\left(c_{D}-c_{L} \alpha_{r} \cos \Lambda\right) q_{\infty} c \sin \Lambda \cos \Lambda \\
f_{y}^{a}=c_{D} q_{\infty} c \cos ^{2} \Lambda \\
f_{z}^{a}=c_{L} q_{\infty} c \cos \Lambda \cos \Gamma \\
m_{x}^{a}=-c_{m} q_{\infty} c^{2} \cos ^{2} \Lambda \cos \Gamma \\
m_{y}^{a}=c_{m} q_{\infty} c^{2} \sin \Lambda \cos \Lambda \\
m_{z}^{a}=c_{m} q_{\infty} c^{2} \cos ^{2} \Lambda \sin \Gamma
\end{gathered}
$$

\section{Aeroelastic Equations for Large Deflection with Axial Force under Pure Rolling Motion}

Consider a special case of pure rolling motion for a highly flexible wing with large flapwise bending deflection. Assuming the effect of flapwise bending is the most dominant, then the inertia, aeroelastic, and elastic forces and moment are considered as follows:

\section{A. Inertial Forces and Moment}

Ignoring the inertial force coupling with the axial displacement and chordwise bending, the inertial forces and moment for pure rolling motion are obtained as

$$
\begin{gathered}
f_{x}^{i}=\rho A\left[-x_{t t}+y_{t} \omega_{z}-z_{t} \omega_{y}-\left(\dot{\omega}_{y}+\omega_{x} \omega_{z}\right) W-2 \omega_{y} W_{t}+z_{t} W_{x t}-U_{t t}\right]+\rho A e_{c g}\left[-\left(\dot{\omega}_{y}+\omega_{x} \omega_{z}\right) \Theta\right. \\
\left.-2 \omega_{y} \Theta_{t}+V_{x t t}\right]+\Delta f_{x}^{i} \\
\begin{array}{r}
f_{y}^{i}=\rho A\left[-y_{t t}-x_{t} \omega_{z}+z_{t} \omega_{x}+\left(\dot{\omega}_{x}-\omega_{y} \omega_{z}\right) W+2 \omega_{x} W_{t}-V_{t t}\right]+\rho A e_{c g}\left[\left(\dot{\omega}_{x}-\omega_{y} \omega_{z}\right) \Theta+2 \omega_{x} \Theta_{t}\right] \\
+\frac{\partial}{\partial x}\left\{\rho A e_{c g}\left[-x_{t t}+y_{t} \omega_{z}-z_{t} \omega_{y}-\left(\dot{\omega}_{y}+\omega_{x} \omega_{z}\right) W-2 \omega_{y} W_{t}+z_{t} W_{x t}-U_{t t}\right]\right\} \\
+\frac{\partial}{\partial x}\left\{\rho I_{z z}\left[-\left(\dot{\omega}_{y}+\omega_{x} \omega_{z}\right) \Theta-2 \omega_{y} \Theta_{t}+V_{x t t}\right]\right\}+\Delta f_{y}^{i} \\
f_{z}^{i}=\rho A\left[-z_{t t}+x_{t} \omega_{y}-y_{t} \omega_{x}+\left(\omega_{x}^{2}+\omega_{y}^{2}\right) W-y_{t} \Theta_{t}-x_{t} W_{x t}-W_{t t}\right]+\rho A e_{c g}\left[\left(\omega_{x}^{2}+\omega_{y}^{2}\right) \Theta-\Theta_{t t}\right] \\
+\frac{\partial}{\partial x}\left[\rho A\left(-x_{t t} W-x_{t} W_{t}\right)\right]+\frac{\partial}{\partial x}\left[\rho A e_{c g}\left(-x_{t t} \Theta-x_{t} \Theta_{t}\right)\right]+\frac{\partial}{\partial x}\left\{\rho I _ { y y } \left[-\left(\dot{\omega}_{z}-\omega_{x} \omega_{y}\right) \Theta\right.\right. \\
\left.\left.-\left(\omega_{y}^{2}+\omega_{z}^{2}\right) W_{x}-2 \omega_{z} \Theta_{t}+W_{x t t}\right]\right\}+\Delta f_{z}^{i}
\end{array}
\end{gathered}
$$




$$
\begin{array}{r}
m_{x}^{i}=\rho A\left(y_{t t} W+y_{t} W_{t}\right)+\rho A e_{c g}\left[-z_{t t}+x_{t} \omega_{y}-y_{t} \omega_{x}+\left(\omega_{x}^{2}+\omega_{y}^{2}\right) W+y_{t t} \Theta-x_{t} W_{x t}-W_{t t}\right] \\
-\rho I_{x x} \Theta_{t t}+\rho I_{y y}\left[\left(\omega_{x}^{2}+\omega_{z}^{2}\right) \Theta-\left(\dot{\omega}_{z}+\omega_{x} \omega_{y}\right) W_{x}-2 \omega_{z} W_{x t}\right]+\rho I_{z z}\left(\omega_{x}^{2}+\omega_{y}^{2}\right) \Theta+\Delta m_{x}^{i}
\end{array}
$$

where

$$
\begin{gathered}
\Delta f_{x}^{i}=\rho A\left(2 W_{t} W_{x t}+W W_{x t t}\right) \\
\Delta f_{y}^{i}=\rho A \omega_{z} W W_{x t}+\frac{\partial}{\partial x}\left[\rho A e_{c g}\left(2 W_{t} W_{x t}+W W_{x t t}\right)\right]+\frac{\partial}{\partial x}\left[\rho I_{y y}\left(\dot{\omega}_{z} W_{x}^{2}+2 \omega_{z} W_{x} W_{x t}\right)\right] \\
\Delta f_{z}^{i}=-2 \rho A \omega_{y} W W_{x t}+\frac{\partial}{\partial x}\left[\rho A\left(-\dot{\omega}_{y} W^{2}-2 \omega_{y} W W_{t}\right)\right]-\frac{\partial}{\partial x}\left(\rho I_{y y} \dot{\omega}_{y} W_{x}^{2}\right) \\
\Delta m_{x}^{i}=\rho A\left(-\dot{\omega}_{x} W^{2}-2 \omega_{x} W W_{t}\right)-2 \rho A e_{c g} \omega_{y} W W_{x t}+\rho I_{y y} \omega_{x} W_{x} W_{x t}
\end{gathered}
$$

with

$$
\begin{gathered}
{\left[\begin{array}{l}
x_{t} \\
y_{t} \\
z_{t}
\end{array}\right]=\left[\begin{array}{c}
-V_{\infty} \sin \Lambda \cos \Gamma+p y_{a} \sin \Gamma \\
-V_{\infty} \cos \Lambda \\
V_{\infty} \sin \Lambda \sin \Gamma+p y_{a} \cos \Gamma
\end{array}\right]} \\
{\left[\begin{array}{l}
\omega_{x} \\
\omega_{y} \\
\omega_{z}
\end{array}\right]=\left[\begin{array}{c}
-p \sin \Lambda \cos \Gamma \\
-p \cos \Lambda \\
p \sin \Lambda \sin \Gamma
\end{array}\right]}
\end{gathered}
$$

by neglecting $v, w$, and $p z_{a}$ which are assumed to be small and noting that $u \approx V_{\infty}$.

\section{B. Unsteady Lift and Pitching Moment}

The aeroelastic angle of attack is given by

$$
\begin{aligned}
\alpha_{e}(x, y)=\left(\frac{\omega_{y}}{u \cos \Lambda}-\frac{z_{t} \omega_{z}}{u^{2} \cos ^{2} \Lambda}\right) U-\frac{\omega_{x} V}{u \cos \Lambda}+\frac{z_{t} \omega_{x} W}{u^{2} \cos ^{2} \Lambda}+\left[\frac{y_{t}}{u \cos \Lambda}-\frac{z_{t}\left(-\omega_{x} y+z_{t}\right)}{u^{2} \cos ^{2} \Lambda}\right] \Theta \\
\quad+\left(-\frac{\omega_{y} y}{u \cos \Lambda}+\frac{z_{t} x_{t}}{u^{2} \cos ^{2} \Lambda}\right) V_{x}+\frac{x_{t} W_{x}}{u \cos \Lambda}-\frac{z_{t} V_{t}}{u^{2} \cos ^{2} \Lambda}-\frac{W_{t}}{u \cos \Lambda}-\frac{y \Theta_{t}}{u \cos \Lambda}+\frac{\omega_{y} W W_{x}}{u \cos \Lambda}
\end{aligned}
$$

Furthermore if we neglect the contribution of the axial displacement and chordwise bending, then aeroelastic angle of attack is further simplified to

$$
\begin{gathered}
\alpha_{e}(x, y)=\frac{z_{t} \omega_{x} W}{V_{\infty}^{2} \cos ^{2} \Lambda}-\left[1+\frac{z_{t}\left(-\omega_{x} y+z_{t}\right)}{V_{\infty}^{2} \cos ^{2} \Lambda}\right] \Theta+\frac{x_{t} W_{x}}{V_{\infty} \cos \Lambda}-\frac{W_{t}}{V_{\infty} \cos \Lambda}-\frac{y \Theta_{t}}{V_{\infty} \cos \Lambda}+\frac{\omega_{y} W W_{x}}{u \cos \Lambda} \\
\alpha_{2}(x, y)=\frac{z_{t} \omega_{x} y \Theta}{V_{\infty}^{2} \cos ^{2} \Lambda}-\frac{y \Theta_{t}}{V_{\infty} \cos \Lambda}
\end{gathered}
$$

The lift and pitching coefficients can then be computed from $\alpha_{e}(x, y)$ and $\alpha_{2}(x, y)$ as follows:

$$
\begin{array}{r}
c_{L}=c_{L_{\alpha}} \cos \Lambda \alpha_{r}+c_{L_{\alpha}} \cos \Lambda C(k)\left[\frac{z_{t} \omega_{x} W}{V_{\infty}^{2} \cos ^{2} \Lambda}-\left(1+\frac{-z_{t} \omega_{x} e_{c} \cos \Lambda+z_{t}^{2}}{V_{\infty}^{2} \cos ^{2} \Lambda}\right) \Theta+\frac{x_{t} W_{x}}{V_{\infty} \cos \Lambda}\right. \\
\left.-\frac{W_{t}}{V_{\infty} \cos \Lambda}-\frac{e_{c} \Theta_{t}}{V_{\infty}}+\frac{\omega_{y} W W_{x}}{V_{\infty} \cos \Lambda}\right]+\frac{2 \pi \cos \Lambda c}{4 V_{\infty}}\left[\frac{z_{t} \omega_{x} W_{t}}{V_{\infty}^{2} \cos ^{2} \Lambda}-\left(1+\frac{-z_{t} \omega_{x} e_{m} \cos \Lambda+z_{t}^{2}}{V_{\infty}^{2} \cos ^{2} \Lambda}\right) \Theta_{t}\right. \\
\left.+\frac{x_{t} W_{x t}}{V_{\infty} \cos \Lambda}-\frac{W_{t t}}{V_{\infty} \cos \Lambda}-\frac{e_{m} \Theta_{t t}}{V_{\infty}}+\frac{\omega_{y}\left(W_{t} W_{x}+W W_{x t}\right)}{V_{\infty} \cos \Lambda}\right] \\
c_{m}=c_{m_{a c}}+\frac{e}{c} c_{L_{\alpha}} \cos \Lambda \alpha_{r}+\frac{e}{c} c_{L_{\alpha}} \cos \Lambda C(k)\left[\frac{z_{t} \omega_{x} W}{V_{\infty}^{2} \cos ^{2} \Lambda}-\left(1+\frac{-z_{t} \omega_{x} e_{c} \cos \Lambda+z_{t}^{2}}{V_{\infty}^{2} \cos ^{2} \Lambda}\right) \Theta\right. \\
\left.+\frac{x_{t} W_{x}}{V_{\infty} \cos \Lambda}-\frac{W_{t}}{V_{\infty} \cos \Lambda}-\frac{e_{c} \Theta_{t}}{V_{\infty}}+\frac{\omega_{y} W W_{x}}{V_{\infty} \cos \Lambda}\right]+2 \pi \cos \Lambda\left\{-\frac{z_{t} \omega_{x} c}{16 V_{\infty}^{2} \cos \Lambda}-\frac{z_{t} \omega_{x} e_{m} W_{t}}{4 V_{\infty}^{3} \cos { }^{2} \Lambda}\right. \\
\left.+\left[\frac{e_{c}}{4 V_{\infty}}+\frac{-z_{t} \omega_{x}\left(c^{2}+32 e_{m}^{2}\right) \cos \Lambda+32 z_{t}^{2} e_{m}}{128 V_{\infty}^{3} \cos ^{2} \Lambda}\right] \Theta_{t}^{2}-\frac{x_{t} e_{m} W_{x t}}{4 V_{\infty}^{2} \cos \Lambda}+\frac{e_{m} W_{t t}}{4 V_{\infty}^{2} \cos \Lambda}-\frac{\omega_{y} e_{m}\left(W_{t} W_{x}+W W_{x t}\right)}{4 V_{\infty}^{2} \cos \Lambda}\right\}
\end{array}
$$


If the dihedral angle is small and can be neglected, then the lift and pitching moment coefficients become

$$
\begin{aligned}
& c_{L}=c_{L_{\alpha}} \cos \Lambda \alpha_{r}+c_{L_{\alpha}} \cos \Lambda C(k)\left[-\frac{p^{2} y_{a} \tan \Lambda W}{V_{\infty}^{2} \cos \Lambda}-\left(1+\frac{p^{2} y_{a} e_{c} \cos \Lambda \sin \Lambda+p^{2} y_{a}^{2}}{V_{\infty}^{2} \cos ^{2} \Lambda}\right) \Theta-\tan \Lambda W_{x}\right. \\
& \left.-\frac{W_{t}}{V_{\infty} \cos \Lambda}-\frac{e_{c} \Theta_{t}}{V_{\infty}}-\frac{p W W_{x}}{V_{\infty}}\right]+\frac{2 \pi \cos \Lambda c}{4 V_{\infty}}\left[-\frac{p^{2} y_{a} \tan \Lambda W_{t}}{V_{\infty}^{2} \cos \Lambda}-\left(1+\frac{p^{2} y_{a} e_{m} \cos \Lambda \sin \Lambda+p^{2} y_{a}^{2}}{V_{\infty}^{2} \cos ^{2} \Lambda}\right) \Theta_{t}\right. \\
& \left.-\tan \Lambda W_{x t}-\frac{W_{t t}}{V_{\infty} \cos \Lambda}-\frac{e_{m} \Theta_{t t}}{V_{\infty}}-\frac{p\left(W_{t} W_{x}+W W_{x t}\right)}{V_{\infty}}\right] \\
& c_{m}=c_{m_{a c}}+\frac{e}{c} c_{L_{\alpha}} \cos \Lambda \alpha_{r}+\frac{e}{c} c_{L_{\alpha}} \cos \Lambda C(k)\left[-\frac{p^{2} y_{a} \tan \Lambda W}{V_{\infty}^{2} \cos \Lambda}-\left(1+\frac{p^{2} y_{a} e_{c} \cos \Lambda \sin \Lambda+p^{2} y_{a}^{2}}{V_{\infty}^{2} \cos ^{2} \Lambda}\right) \Theta\right. \\
& \left.-\tan \Lambda W_{x}-\frac{W_{t}}{V_{\infty} \cos \Lambda}-\frac{e_{c} \Theta_{t}}{V_{\infty}}-\frac{p W W_{x}}{V_{\infty}}\right]+2 \pi \cos \Lambda\left\{\frac{p^{2} y_{a} c \tan \Lambda \Theta}{16 V_{\infty}^{2}}+\frac{p^{2} y_{a} e_{m} \tan \Lambda W_{t}}{4 V_{\infty}^{3} \cos \Lambda}\right. \\
& +\left[\frac{e_{c}}{4 V_{\infty}}+\frac{p^{2} y_{a}\left(c^{2}+32 e_{m}^{2}\right) \cos \Lambda \sin \Lambda+32 p^{2} y_{a}^{2} e_{m}}{128 V_{\infty}^{3} \cos ^{2} \Lambda}\right] \Theta_{t}+\frac{e_{m} \tan \Lambda W_{x t}}{4 V_{\infty}}+\frac{e_{m} W_{t t}}{4 V_{\infty}^{2} \cos \Lambda} \\
& \left.+\frac{\left(c^{2}+32 e_{m}^{2}\right) \Theta_{t t}}{128 V_{\infty}^{2}}+\frac{p e_{m}\left(W_{t} W_{x}+W W_{x t}\right)}{4 V_{\infty}^{2}}\right\}
\end{aligned}
$$

\section{Elastic Forces and Moment}

Retaining only the nonlinear terms with $U_{x}$ and $W_{x}$, and assuming that $e_{a}$ is small, then the elastic forces and moment are given by

$$
\left[\begin{array}{c}
P_{x} \\
M_{x} \\
M_{y} \\
M_{z}
\end{array}\right]=\left[\begin{array}{c}
E A U_{x}+\frac{1}{2} E A U_{x}^{2}+\frac{1}{2} E A W_{x}^{2}+\frac{1}{2} E I_{y y} W_{x x}^{2} \\
\left(G J+E I_{x x} U_{x}+\frac{1}{2} E I_{x x} U_{x}^{2}+\frac{1}{2} E I_{x x} W_{x}^{2}+\frac{1}{2} E B_{5} W_{x x}^{2}\right) \Theta_{x} \\
\left(1+U_{x}\right) E I_{y y} W_{x x} \\
\left(1+U_{x}\right) E I_{z z} V_{x x}-\frac{1}{2} E B_{9} W_{x x}^{2}
\end{array}\right]
$$

The resulting equilibrium equations are given by ${ }^{8}$

$$
\begin{gathered}
\frac{\partial}{\partial x}\left[\begin{array}{c}
P_{x} \\
P_{y} \\
P_{z}
\end{array}\right]=-\left[\begin{array}{c}
f_{x} \\
f_{y} \\
f_{z}
\end{array}\right] \\
\frac{\partial}{\partial x}\left[\begin{array}{c}
M_{x} \\
M_{y} \\
M_{z}
\end{array}\right]=\left[\begin{array}{c}
P_{y} W_{x}-P_{z} V_{x}-m_{x} \\
P_{x} W_{x}-P_{z}+m_{y} \\
P_{x} V_{x}-P_{y}-m_{z}
\end{array}\right]
\end{gathered}
$$

These equations become

$$
\begin{aligned}
\frac{\partial M_{x}}{\partial x}+\left(\frac{\partial M_{z}}{\partial x}-\right. & \left.P_{x} V_{x}+m_{z}\right) W_{x}-\left(\frac{\partial M_{y}}{\partial x}-P_{x} W_{x}-m_{y}\right) V_{x}+m_{x}=0 \\
& \frac{\partial^{2} M_{y}}{\partial x^{2}}-\frac{\partial\left(P_{x} W_{x}\right)}{\partial x}-\frac{\partial m_{y}}{\partial x}-f_{z}=0 \\
& \frac{\partial^{2} M_{z}}{\partial x^{2}}-\frac{\partial\left(P_{x} V_{x}\right)}{\partial x}+\frac{\partial m_{z}}{\partial x}-f_{y}=0
\end{aligned}
$$

Neglecting the nonlinear terms with $V_{x}$, the nonlinear equations are obtained as

$$
\frac{\partial}{\partial x}\left(E A U_{x}+\frac{1}{2} E A U_{x}^{2}+\frac{1}{2} E A W_{x}^{2}+\frac{1}{2} E I_{y y} W_{x x}^{2}\right)=-f_{x}
$$




$$
\begin{gathered}
\frac{\partial}{\partial x}\left[\left(G J+E I_{x x} U_{x}+\frac{1}{2} E I_{x x} U_{x}^{2}+\frac{1}{2} E I_{x x} W_{x}^{2}+\frac{1}{2} E B_{5} W_{x x}^{2}\right) \Theta_{x}\right]=-m_{x} \\
\frac{\partial^{2}\left[\left(1+U_{x}\right) E I_{y y} W_{x x}\right]}{\partial x^{2}}-\frac{\partial\left(P_{x} W_{x}\right)}{\partial x}=f_{z}+\frac{\partial m_{y}}{\partial x} \\
\frac{\partial^{2}\left[\left(1+U_{x}\right) E I_{z z} V_{x x}-\frac{1}{2} E B_{9} W_{x x}^{2}\right]}{\partial x^{2}}-\frac{\partial\left(P_{x} V_{x}\right)}{\partial x}=f_{y}-\frac{\partial m_{z}}{\partial x}
\end{gathered}
$$

where $P_{x}=E A U_{x}+\frac{1}{2} E A U_{x}^{2}+\frac{1}{2} E A W_{x}^{2}+\frac{1}{2} E I_{y y} W_{x x}^{2}$.

Consider a weak-form solution by letting $U(x, t)=N_{u}(x) u(t), V(x, t)=N_{v}(x) v, W(x, t)=N_{w}(x) w(t)$, and $\Theta(x, t)=N_{\theta}(x) \theta(t)$. Then we obtain the following weak-form expressions

$$
\begin{aligned}
& u \int_{0}^{L} N_{u}^{\prime \top} E A N_{u} d x+\frac{1}{2} u^{2} \int_{0}^{L} N_{u}^{\prime \top}\left(E A N_{u}^{\prime}\right) N_{x}^{\prime} d x+\frac{1}{2} w^{2} \int_{0}^{L} N_{u}^{\prime \top}\left(E A N_{w}^{\prime}\right) N_{w}^{\prime} d x \\
& +\frac{1}{2} w^{2} \int_{0}^{L} N_{u}^{\prime \top}\left(E I_{y y} N_{w}^{\prime \prime}\right) N_{w}^{\prime \prime} d x=\int_{0}^{L} N_{u}^{\top} f_{x} d x \\
& \theta \int_{0}^{L} N_{\theta}^{\prime \top} G J N_{\theta}^{\prime} d x+\theta u \int_{0}^{L} N_{\theta}^{\prime \top}\left(E I_{x x} N_{u}^{\prime}\right) N_{\theta}^{\prime} d x+\frac{1}{2} \theta u^{2} \int_{0}^{L} N_{\theta}^{\prime \top}\left(E I_{x x} N_{u}^{\prime 2}\right) N_{\theta}^{\prime} d x \\
& +\frac{1}{2} \theta w^{2} \int_{0}^{L} N_{\theta}^{\prime \top}\left(E I_{x x} N_{w}^{\prime 2}\right) N_{\theta}^{\prime} d x+\frac{1}{2} \theta w^{2} \int_{0}^{L} N_{\theta}^{\prime \top}\left(E B_{5} N_{w}^{\prime \prime 2}\right) N_{\theta}^{\prime} d x=\int_{0}^{L} N_{\theta}^{\top} m_{x} d x \\
& w \int_{0}^{L} N_{w}^{\prime \prime} \top E I_{y y} N_{w}^{\prime \prime} d x+w u \int_{0}^{L} N_{w}^{\prime \prime} \top\left(E I_{y y} N_{u}^{\prime}\right) N_{w}^{\prime \prime} d x+w u \int_{0}^{L} N_{w}^{\prime \top}\left(E A N_{u}^{\prime}\right) N_{w}^{\prime} d x \\
& +\frac{1}{2} w u^{2} \int_{0}^{L} N_{w}^{\prime \top}\left(E A N_{u}^{\prime 2}\right) N_{w}^{\prime} d x+\frac{1}{2} w^{3} \int_{0}^{L} N_{w}^{\prime \top}\left(E A N_{w}^{\prime 2}\right) N_{w}^{\prime} d x \\
& +\frac{1}{2} w^{3} \int_{0}^{L} N_{w}^{\prime}\left(E I_{y y} N_{w}^{\prime \prime 2}\right) N_{w}^{\prime} d x=\int_{0}^{L} N_{w}^{\top}\left(f_{z}+\frac{\partial m_{y}}{\partial x}\right) d x \\
& v \int_{0}^{L} N_{v}^{\prime \prime \top} E I_{z z} N_{v}^{\prime \prime} d x+v u \int_{0}^{L} N_{v}^{\prime \prime} \top\left(E I_{z z} N_{u}^{\prime}\right) N_{v}^{\prime \prime} d x-\frac{1}{2} w^{2} \int_{0}^{L} N_{v}^{\prime \prime} \top\left(E B_{9} N_{w}^{\prime \prime}\right) N_{w}^{\prime \prime} \\
& +v u \int_{0}^{L} N_{v}^{\prime \top}\left(E A N_{u}^{\prime}\right) N_{v}^{\prime} d x+\frac{1}{2} v u^{2} \int_{0}^{L} N_{v}^{\prime \top}\left(E A N_{u}^{\prime 2}\right) N_{v}^{\prime} d x \\
& +\frac{1}{2} v w^{2} \int_{0}^{L} N_{v}^{\prime \top}\left(E A N_{w}^{\prime 2}\right) N_{v}^{\prime} d x+\frac{1}{2} v w^{2} \int_{0}^{L} N_{v}^{\prime \top}\left(E I_{y y} N_{w}^{\prime \prime 2}\right) N_{v}^{\prime} d x=\int_{0}^{L} N_{w}^{\top}\left(f_{y}-\frac{\partial m_{z}}{\partial x}\right) d x
\end{aligned}
$$

These equations constitute a set of nonlinear displacement equations which can be solved by any nonlinear root search methods.

For illustration, consider a cantilever wing with the bending stiffness $E I=2 \times 10^{9} e^{-7 \eta}$ and extensional stiffness $E A=9.5806 \times 10^{6} \eta^{2}-24.1861 \times 10^{6} \eta+18.0679 \times 10^{6}$ where $\eta=\frac{x}{L}$. The wing tip deflection is $6.21 \%$ of the wing length with the linear analysis, and is $6.03 \%$ with the nonlinear analysis. This wing tip deflection is similar to that of a conventional aluminum wing such as the Boeing 757 wing. As the bending stiffness reduces, the effect of nonlinear bending becomes more pronounced as the contribution of the nonlinear bending stiffness due to the wing bending slope $W_{x}$ causes the aeroelastic deflection to decrease relative to the linear aeroelastic deflection. At about $10 \%$ wing tip deflection, the effect of nonlinear bending begins to set in. This wing tip deflection is similar to that of a modern composite wing such as the Boeing 787 wing. Figure 2 illustrates the nonlinear bending of a cantilever wing. 


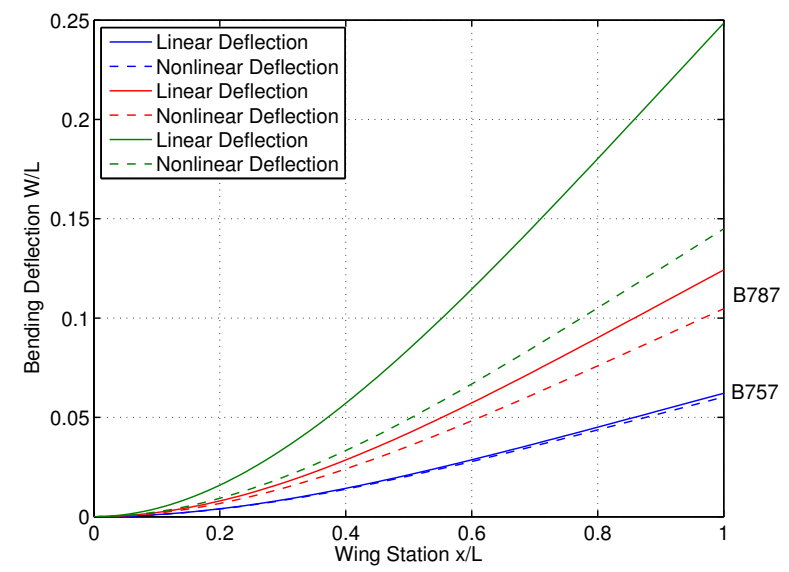

Figure 2. Nonlinear Bending of Typical Cantilever Wing

\section{Aeroelastic Analysis of Truss-Braced Wing Aircraft}

The SUGAR Truss-Braced Wing (TBW) aircraft concept is a Boeing-developed N+3 aircraft configuration funded by NASA ARMD Fixed Wing project. ${ }^{12-14}$ The TBW aircraft concept is designed to be aerodynamically efficient by employing a high-aspect ratio wing design. The aspect ratio of the TBW is in the order of 14 which is significantly greater than those of conventional aircraft wings. As a result, intermediate structural supports are required. The main wings are braced at approximately mid-span by two main struts. In addition, two jury struts; one on each wing, provide additional reinforcement. Figure 3 is an illustration of the TBW aircraft.

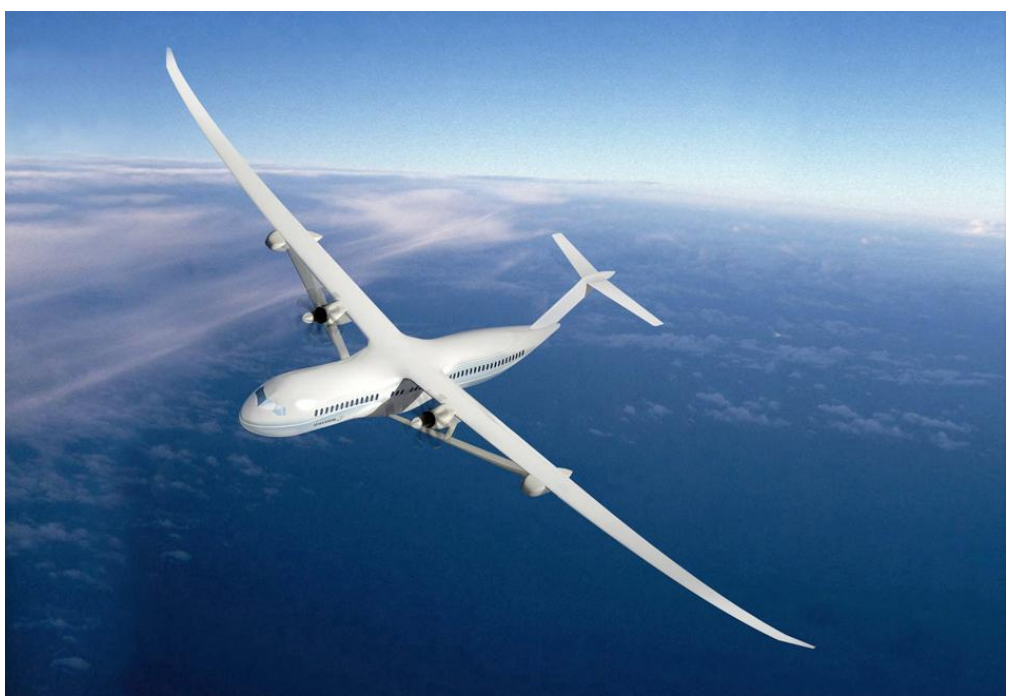

Figure 3. Boeing SUGAR Truss-Braced Wing (TBW) Aircraft Concept

Truss-braced wing aircraft concepts provide a structural solution to high aspect ratio wing aircraft designs. The long slender wing would employ structural bracing via the use of axially loaded strut members to provide intermediate span supports in addition to the wing root attachment. These struts generally support a portion of the span load carried by the wing and are loaded in tension. Under a negative-g flight condition such as during a dive, a load reversal could occur that could put the struts in compression. The compressive loading would require design considerations for buckling strength.

Under aerodynamic loading, an axially loaded member also experiences the normal bending and torsion generated by aerodynamic lift force and pitching moment. Aeroelasticity of an axially loaded structure 
undergoing transverse bending can be significantly different from that with transverse bending alone. The main struts and jury struts can impact aerodynamics of the main wings since they create aerodynamic interference drag that can offset the aerodynamic benefit of high-aspect ratio wing design. Thus, the TBW is designed to strive a balance between aerodynamic efficiency and structural efficiency.

A recent flutter wind tunnel test of a dynamically scaled TBW was conducted in NASA Langley Transonic Dynamic Wind Tunnel (TDT). ${ }^{15}$ The model is $16 \%$ scaled, semi-span, wall-mounted model as shown in Fig. 4. It was reported that the flutter speed is dependent on the angle of attack at $\pm 1^{\circ} .{ }^{15}$ Test data shows that the flutter boundary for the TBW is generally lower at $-1^{\circ}$ than at $+1^{\circ}$ in the angle of attack. The nonlinear effect of the TBW due to axial loading has been analyzed. ${ }^{16,17}$ While there may be other nonlinear factors that could contribute to this observation, one plausible explanation could be the geometric nonlinear tension stiffening effect of the main struts at a positive angle of attack, and conversely the softening effect at a negative angle of attack.

A finite-element model (FEM) of the TBW is constructed that uses a total of five components. Only the right side of the aircraft is modeled, and the wing configuration is assumed to be cantilevered to the side of the fuselage. The three main physical components of the wing configuration are the wing, the strut, and the jury strut. The FEM uses lumped mass and inertia properties provided by Boeing Research \& Technology. ${ }^{13}$ The lumped mass data is converted into the running mass. Extensional stiffness of the wing is not available and instead is estimated for the purpose of illustrating the nonlinear effects. Since the nonlinear effect is sensitive to the extensional stiffness, the solution can vary widely depending on the extensional stiffness. For this study, we choose the extensional stiffness $E A=3$ The engine-pylon mass is not available and therefore is not modeled. It should be noted that the model is not an accurate representation of the Boeing TBW model, but instead is intended for illustrative purposes only to demonstrate the nonlinear aeroelasticity with inertial force coupling.

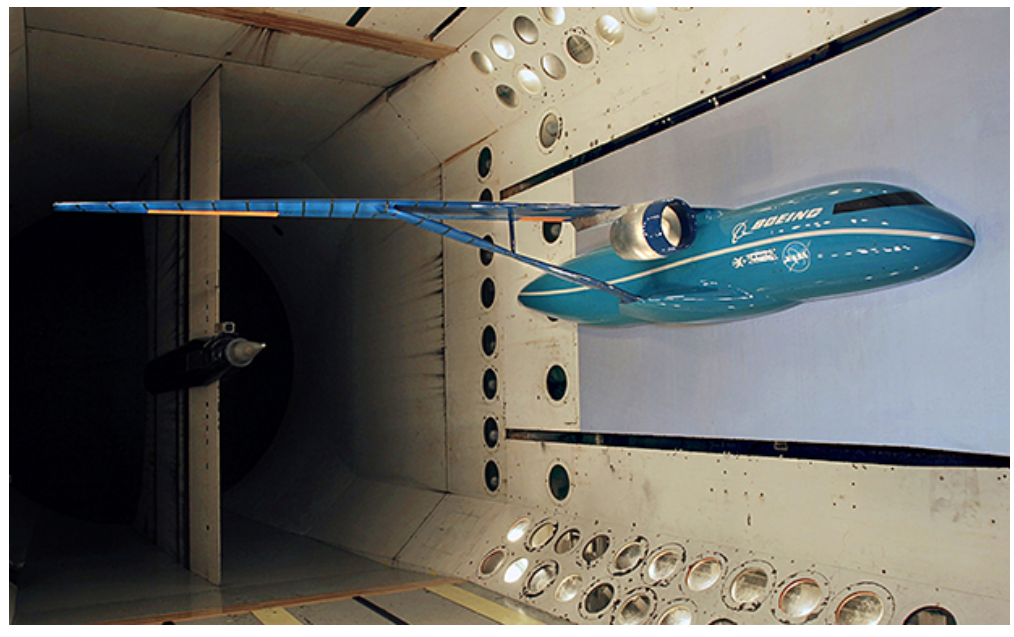

Figure 4. Truss-Braced Wing (TBW) Model in NASA Langley Transonic Dynamic Tunnel (TDT)

Figures 5(a) and (b) are the plots of the lift and pitching moment distributions for the wing and the strut at 2.5-g condition, corresponding to $C_{L}=2.025$ at Mach 0.7 and an altitude of $42,000 \mathrm{ft}$ for a design cruise gross weight of $146,000 \mathrm{lbs}$ based on a wing reference area of $1,477 \mathrm{ft}^{2}$. 


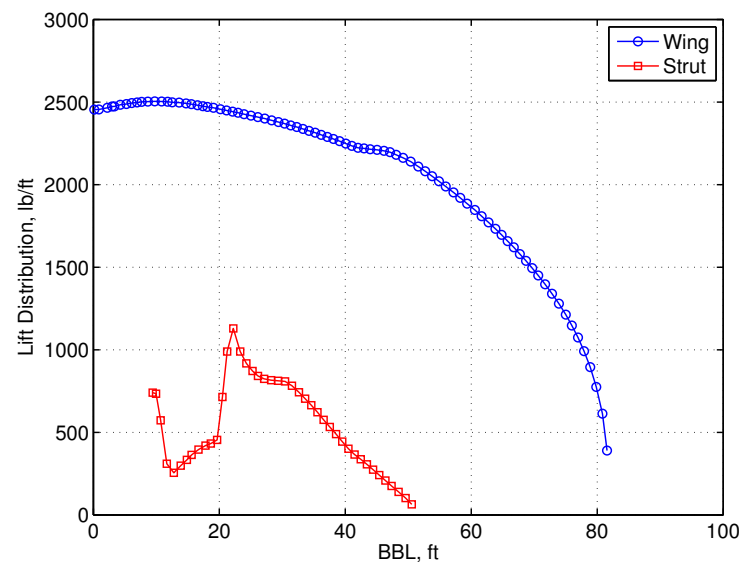

(a)

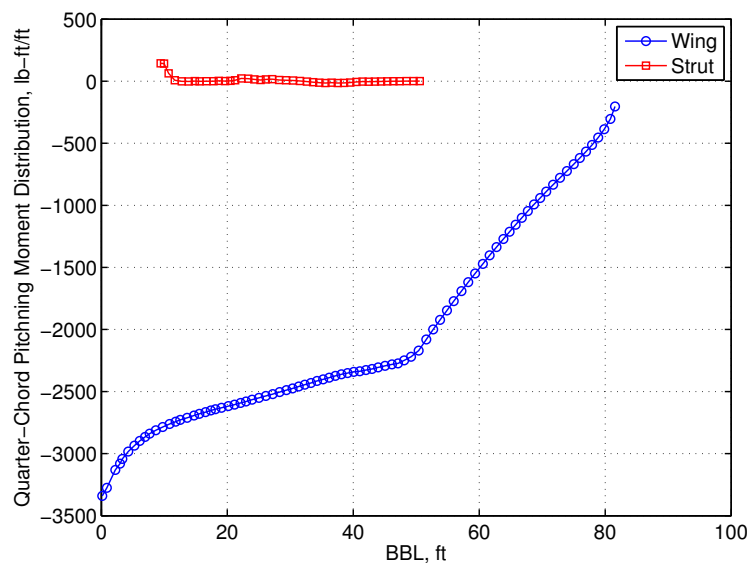

(b)

Figure 5. Lift and Pitching Moment Distribution at $2.5 \mathrm{~g}$

Figure 6 is the plot of the wing vertical deflection for linear and nonlinear analyses. At a 2.5-g flight load with no inertial force coupling and nonlinear tension-induced stiffness due to the strut, the vertical deflection of the wing tip is computed to be $3.1009 \mathrm{ft}$. With the tension-induced stiffness, the vertical deflection is reduced to $3.0765 \mathrm{ft}$. The effect of the nonlinear tension-induced stiffness is apparently not significant. When the nonlinear bending stiffness is accounted for, the vertical deflection is significantly reduced to a value of $2.2930 \mathrm{ft}$. Note that the linear vertical deflection is about $9 \%$ of the wing span from the strut to the wing tip. Thus, it is not unreasonable to expect that nonlinear bending effect begins to set in at this value of bending deflection.

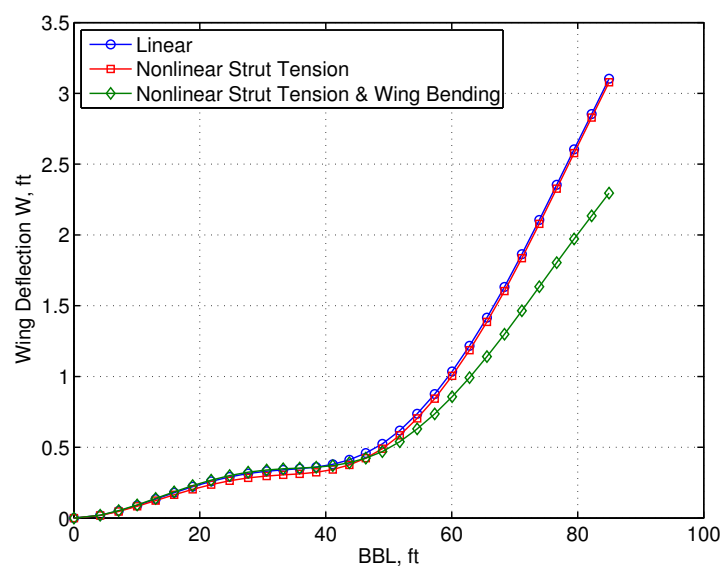

Figure 6. Wing Vertical Deflection at 2.5-g Flight Condition

Figure 7 shows the effect of the roll rate on the wing tip deflection for linear and nonlinear analyses. As the roll rate increases, the wing tip deflection increases nonlinearly with the roll rate. The inertial force coupling of roll rate is reduced by the nonlinear tension-induced and large bending deflection. For typical transport aircraft, the maximum roll rate may be limited to about $20 \mathrm{deg} / \mathrm{sec}$. Thus, the inertial force coupling for the TBW is not considered to be significant. However, in general, for high aspect ratio wing aircraft, the inertial force coupling can be important. 


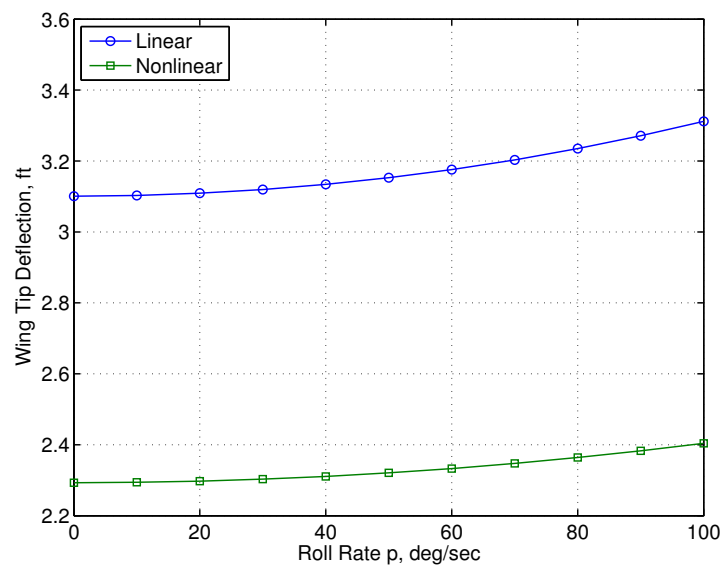

Figure 7. Wing Tip Vertical Deflection at 2.5-g Flight Condition as Function of Roll Rate

Table 2 shows the frequencies for linear analysis and nonlinear analysis with and without inertial force coupling. The nonlinear effect of strut tension stiffening and large bending deflection contributes to the increase in the wing stiffness results in the frequency increase. For a roll rate of $20 \mathrm{deg} / \mathrm{sec}$, the frequencies only change very slightly.

\begin{tabular}{|c|c|c|c|}
\hline Mode & Linear & Nonlinear & Nonlinear with 20-deg/sec Roll Rate \\
\hline \hline 1 & 1.9418 & 2.2311 & 2.2311 \\
\hline 2 & 2.3631 & 2.7418 & 2.7420 \\
\hline 3 & 4.0649 & 4.3267 & 4.3266 \\
\hline 4 & 6.7069 & 7.0629 & 7.0632 \\
\hline 5 & 8.5323 & 8.7955 & 8.7068 \\
\hline
\end{tabular}

Table 2. Frequencies of TBW at 2.5-g Flight Condition

Figures 8(a) and (b) show the frequency and damping at 2.5-g flight condition for linear analysis. As can be seen in Fig. 8(a), the flutter speed occurs at 606 KEAS (Knot Equivalent Air Speed) corresponding to mode 5 at 2.5-g. For nonlinear analysis, the frequency and damping at 2.5-g flight condition are plotted in Figs. 9(a) and (b). Due to the nonlinear stiffness, the flutter speed increases to $643 \mathrm{KEAS}$.

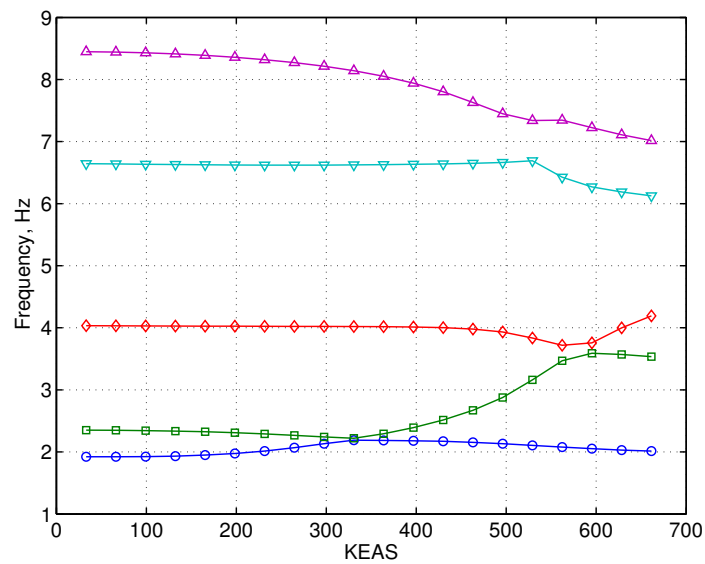

(a)

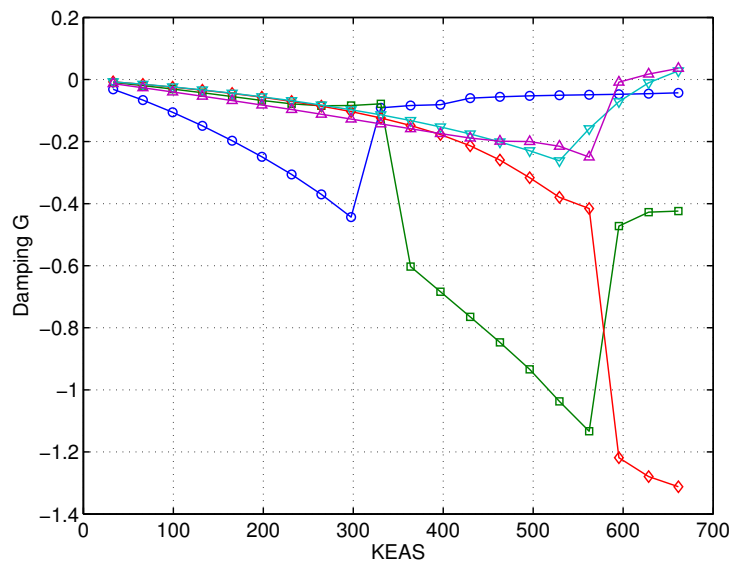

(b)

Figure 8. Frequency and Damping at 2.5-g Flight Condition with Linear Analysis 


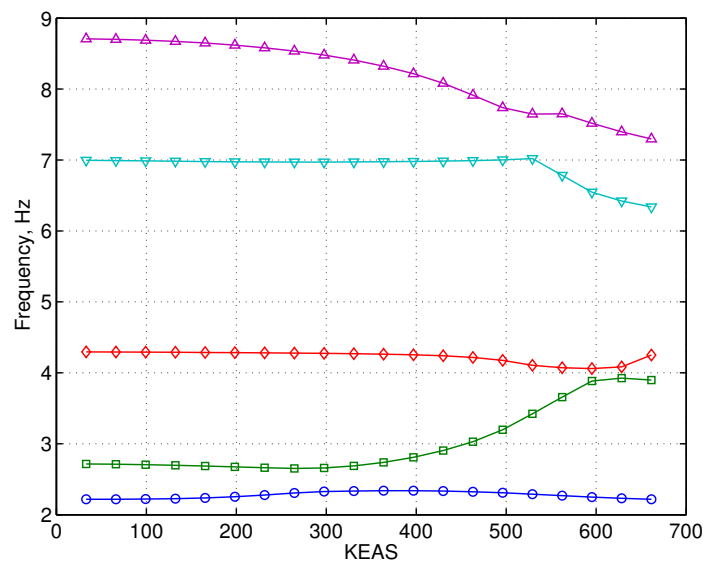

(a)

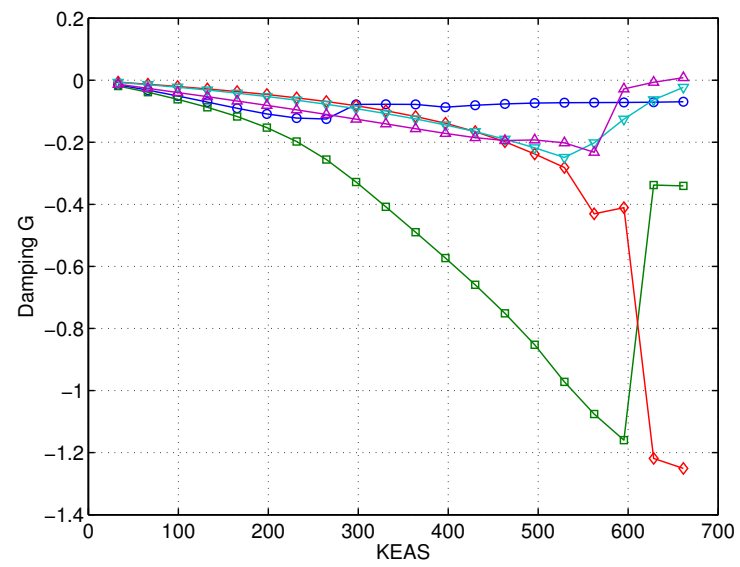

(b)

Figure 9. Frequency and Damping at 2.5-g Flight Condition with Nonlinear Analysis

It should be noted that flutter is a linear aeroelasticity concept. Nonlinear aeroelasticity can give rise to limit cycle oscillations which can be analyzed more conveniently in the time domain than in the frequency domain. As future aircraft tend to employ higher-aspect ratio wing design, nonlinear aeroelasticity can become increasingly more important that will require a full nonlinear aeroelastic consideration with inertial force coupling in the initial design. Future studies will investigate the effects of large deflection on a different high aspect ratio wing aircraft model such as the Common Research Model (CRM) or by redesigning the TBW aircraft to allow larger wing deflections by reducing the effect of the strut tension.

\section{Conclusions}

This paper presents an analytical method for coupling inertial forces into wing aeroelasticity. The inertial forces resulting from aircraft angular rate and acceleration interact with wing aeroelasticity in a complex manner. If aeroelastic deflections are significant, the inertial force coupling effect can introduce nonlinearity in the aeroelastic analysis. The Theodorsen's method for unsteady aerodynamics is applied to incorporate the inertial force coupling into the formulation of the aeroelastic angle of attack. Additionally, the elastic forces are moments are formulated to account for large flapwise bending. This results in a set of nonlinear aeroelastic weak-form equations. An analysis of the Truss-Braced Wing (TBW) aircraft is performed for a pure rolling motion. The analysis at 2.5-g flight loads demonstrates that the inertial force coupling causes a small increase in the wing deflection. The nonlinear tension-induced stiffness due to the strut causes a slight reduction in the wing deflection. On the other hand, the nonlinear bending effect can be significant if the wing tip bending deflection is sufficiently large. The flutter speed is shown to be highly influenced by the nonlinear bending effect which causes an increase in the flutter speed for the TBW aircraft. In practice, nonlinear bending can give rise to limit cycle oscillations which can be investigated more expediently using a time-domain analysis.

\section{Acknowledgment}

The authors would like to thank the Advanced Air Transport Technology Project under the Fundamental Aeronautics Program of NASA Aeronautics Research Mission Directorate (ARMD) for funding support of this work. The authors also would like to acknowledge Boeing Research and Technology for providing the Truss-Braced Wing aircraft models.

\section{References}

${ }^{1}$ Noll, T. E. and Eastep, F. E., "Active Flexible Wing Program," AIAA Journal of Aircraft, Vol. 32, No. 1, pp. 9-9, 1999.

${ }^{2}$ Pendleton, E., Flick, P., Voracek, D., Reichenbach, E., Griffin, K., and Paul, D., "The X-53,A Summary of the Active 
Aeroelastic Wing Flight Research Program," AIAA Structures, Structural Dynamics, and Materials Conference, AIAA-20071855, Honolulu, HI, April, 2007.

${ }^{3}$ Nguyen, N., "Elastically Shaped Future Air Vehicle Concept," NASA Innovation Fund Award 2010 Report, October 2010, Submitted to NASA Innovative Partnerships Program, http://ntrs.nasa.gov/archive/nasa/casi.ntrs.nasa.gov/20110023698.pdf.

${ }^{4}$ Nguyen, N., Trinh, K., Reynolds, K., Kless, J., Aftosmis, M., Urnes, J., and Ippolito, C., "Elastically Shaped Wing Optimization and Aircraft Concept for Improved Cruise Efficiency," 51st AIAA Aerospace Sciences Meeting, AIAA-2013-0141, January 2013.

${ }^{5}$ Urnes, J., Nguyen, N., Ippolito, C., Totah, J., Trinh, K., and Ting, E., "A Mission Adaptive Variable Camber Flap Control System to Optimize High Lift and Cruise Lift to Drag Ratios of Future N+3 Transport Aircraft," 51st AIAA Aerospace Sciences Meeting, AIAA-2013-0214, January 2013.

${ }^{6}$ Nguyen, N. and Urnes, J., "Aeroelastic Modeling of Elastically Shaped Aircraft Concept via Wing Shaping Control for Drag Reduction," AIAA Atmospheric Flight Mechanics Conference, AIAA-2012-4642, August 2012.

${ }^{7}$ Nguyen, N., "Integrated Flight Dynamics Modeling of Flexible Aircraft with Inertial Force-Propulsion - Aeroelastic Couplings," 46th AIAA Aerospace Sciences Meeting and Exhibit, AIAA-2008-0194, Reno, NV, January 2008.

${ }^{8}$ Houbolt, J. C. and Brooks, G. W., "Differential Equations of Motion for Combined Flapwise Bending, Chordwise Bending, and Torsion of Twisted Nonuniform Rotor Blades," NACA Technical Note 3905, February 1957.

${ }^{9}$ Nguyen, N., Ting, E., Nguyen, D., Dao, T., and Trinh, K., "Coupled Vortex-Lattice Flight Dynamic Model with Aeroelastic Finite-Element Model of Flexible Wing Transport Aircraft with Variable Camber Continuous Trailing Edge Flap for Drag Reduction," AIAA Atmospheric Flight Mechanics, AIAA-2013-4746, August 2013.

${ }^{10}$ Theodorsen, T., "General Theory of Aerodynamic Instability and the Mechanism of Flutter," NACA Report 496, 1949.

${ }^{11}$ Bisplinghoff, R. L., Ashley, H., and Halman, R. L., Aeroelasticity, Addison-Wesley Publishing Company, Inc., 1955.

${ }^{12}$ Bradley, M. K. and Droney, C. K., "Subsonic Ultra Green Aircraft Research: Truss Braced Wing Design Exploration," Contractor Report, The Boeing Company, June 2014.

${ }^{13}$ Bradley, M. K., Droney, C. K., and Allen, T. J., "Subsonic Ultra Green Aircraft Research Phase II - Truss Braced Wing Design Exploration" Contractor Report, The Boeing Company, June 2014.

${ }^{14}$ Bradley, M. K., Droney, C. K., and Allen, T. J., "Subsonic Ultra Green Aircraft Research: Truss Braced Wing Aeroelastic Test Report," Contractor Report, The Boeing Company, June 2014.

${ }^{15}$ Bartels, E. R., Scott, R. C., Funk, C., J., Allen, T., J., and Sexton, B. W., "Comparisons of Computed and Experimental Aeroelastic Stability for the Boeing Truss-Braced Wing Wind Tunnel Model," 32nd AIAA Applied Aerodynamics Conference, AIAA-2014-2446, Atlanta, GA, June 2014.

${ }^{16}$ Coggin, J. M., Kapania, R. K., Zhao, W., Schetz, J. A., Hodigere-Siddaramaiah, V., Allen, T. J., and Sexton, B. W., "Nonlinear Aeroelastic Analysis of a Truss Braced Wing Aircraft," 55th AIAA/ASME/ASCE/AHS/ASC Structures, Structural Dynamics, and Materials Conference, AIAA-2014-0335, National Harbor, MD, January 2014.

${ }^{17}$ Nguyen, N., Ting, E., and Lebofsky, S., "Aeroelasticity of Axially Loaded Aerodynamic Structures for Truss-Braced Wing Aircraft," 56th AIAA/ASME/ASCE/AHS/ASC Structures, Structural Dynamics, and Materials Conference, AIAA-2015-1840, January 2015.

${ }^{18}$ Hodges, D. H. and Pierce, G.A., Introduction to Structural Dynamics and Aeroelasticity, Cambridge University Press, 2002. 